\title{
Metrics of High Cofluctuation and Entropy to Describe Control of Cardiac Function in the Stellate Ganglion
}

Nil Z. Gurel, $\mathrm{PhD}^{1,2^{*}+}$, Koustubh B. Sudarshan, $\mathrm{MS}^{3^{*}}$, Joseph Hadaya, MD ${ }^{1,2,4}$, Alex Karavos, $\mathrm{BS}^{3}$, Taro Temma, MD, PhD ${ }^{1,2}$, Yuichi Hori, $\mathrm{MD}^{1,2}$, J. Andrew Armour, MD, PhD ${ }^{1,2}$, Guy Kember, $\mathrm{PhD}^{3}$, Olujimi A. Ajijola, MD, $\mathrm{PhD}^{1,2,4}$

${ }^{1}$ UCLA Neurocardiology Research Program of Excellence, Los Angeles, CA

${ }^{2}$ UCLA Cardiac Arrhythmia Center, UCLA Health System, Los Angeles, CA

${ }^{3}$ Department of Engineering Mathematics and Internetworking, Dalhousie University, Halifax, Nova Scotia

${ }^{4}$ Molecular, Cellular and Integrative Physiology Program, UCLA, Los Angeles, CA

${ }^{*}$ Co-first authors

Running Head: Cofluctuation and Entropy in Cardiac Neural Control

Keywords: cardiac control; stellate ganglion; neural recordings; neurocardiology; neural population dynamics

Disclosures: University of California, Los Angeles has patents relating to cardiac neural diagnostics and therapeutics. Dr. Ajijola is a co-founder of NeuCures, Inc. The remaining authors have no additional disclosures to report.

Address for correspondence:

Olujimi A. Ajijola MD PhD

UCLA Cardiac Arrhythmia Center

UCLA Neurocardiology Research Center

David Geffen School of Medicine at UCLA.

100 Medical Plaza, Suite 660, Westwood Blvd

Los Angeles CA 90095-1679

Phone: 3102066433 ; Fax: 3108252092

e-mail: oajijola@mednet.ucla.edu

Acknowledgments: This work was funded by the National Institutes of Health, Office of The Director DP2 OD024323-01, and U01EB025138. Nil Z. Gurel was funded by the NSF Engineering Fellows Postdoctoral Fellowship Award ID \#2127509. 


\begin{abstract}
Neural control of the heart involves dynamic adaptation of mechanical and electrical indices to meet blood flow demands. The control system receives centrally-derived inputs to coordinate cardiac function on a beat-by-beat basis, producing "functional" outputs such as the blood pressure waveform. Bilateral stellate ganglia (SG) are responsible for integration of multiple inputs and efferent cardiopulmonary sympathetic neurotransmission. In this work, we investigate network processing of cardiopulmonary transduction by SG neuronal populations in porcine with chronic pacing-induced heart failure and control subjects. We derive novel metrics to describe control of cardiac function by the SG during baseline and stressed states from in vivo extracellular microelectrode recordings. Network-level spatiotemporal dynamic signatures are found by quantifying state changes in coactive neuronal populations (i.e., cofluctuations). Differences in "neural specificity" of SG network activity to specific phases of the cardiac cycle are studied using entropy estimation. Fundamental differences in information processing and cardiac control are evident in chronic heart failure where the SG exhibits: i) short-lived, high amplitude cofluctuations in baseline states, ii) greater variation in neural specificity to cardiac cycles, iii) limited sympathetic reserve during stressed states, and iv) neural network activity and cardiac control linkage that depends on disease state and cofluctuation magnitude. These findings indicate that spatiotemporal dynamics of stellate ganglion neuronal populations are altered in heart failure, and lay the groundwork for understanding dysfunction neuronal signaling reflective of cardiac sympathoexcitation.
\end{abstract}




\section{Introduction}

Neural control of cardiac function involves adaptive adjustment of mechanical and electrical activity to meet the organism's demand for blood flow. This cardioneural control schemeconsists of neural populations in the central, peripheral, and intrinsic cardiac nervous systems. Interactions among components of the cardiac nervous system highlight that these neural populations work in concert, rather than as independent, singular processing units [1]. From an information processing standpoint, the operation of these interconnected neural networks has been evolved to coordinate cardiac function on a beat-by-beat basis, producing the "functional" outputs of this control scheme such as electrocardiogram, blood pressure waveform, or respiratory pressure. Localized adaptations in the cardioneural network in response to pathology can cause an evolution of global network properties toward control with heightened risk of poor outcomes without measurable evidence from these functional outputs [2, 3].

There is a current focus on understanding cardioneural network processing within the stellate ganglion (SG), the major source of sympathetic input to the heart. The SG (located in either side of the neck) operates as an integrative layer within the control hierarchy where it processes central cardiac inputs to the heart, receives cardiac feedback, and projects efferent control outputs to the heart. In pathological states such as heart failure, morphological and neurochemical remodeling of SG neurons has been reported in both animal models [4-7] and in humans $[8,9]$. Due to its key role in proarrhythmic neural signaling and convenience in surgical accessibility, clinical interventions targeting SG are used to treat various cardiovascular conditions [10-12]. It has also been established that an enhanced cardiac sympathetic afferent reflex contributes to sympathoexcitation and pathogenesis of heart failure [13-20]. Despite these novel interventions and general understanding, SG clinical therapy will remain largely 
unexplored without greatly improved understanding of SG neuronal information processing in healthy versus pathological states. Prior studies examining the SG neural activity have been limited to in vivo extracellular recordings [21-25].

Recently, we explored network processing of cardiopulmonary transduction by SG neuronal populations in healthy porcine, defining a novel metric that links neural firing patterns to cardiopulmonary signals [26]. In the current work, we investigate the pathological differences in information transfer in swine models with multi-site electrode arrays. We first uncover networklevel spatiotemporal dynamic signatures by quantifying the state changes in coactive neuronal populations (i.e., cofluctuations) by analyzing continuous time series of neural activity. Second, we study the differences in neural specificity to cardiopulmonary control targets by dynamic non-linear measures based on entropy estimation. We further present how these indices differ in swine models with healthy hearts and swine models that underwent pacemaker-induced heart failure remodeling.

\section{Methods}

\section{Animal Experiments}

Figure 1 presents an overview of study design and data acquisition. The study was performed under a protocol approved by the University of California Los Angeles (UCLA) Animal Research Committee (ARC), in compliance with the UCLA Institutional Animal Care and Use Committee (IACUC) guidelines and the National Institutes of Health (NIH) Guide for the Care and Use of Laboratory Animals. Figure 1D-E summarizes the studied animal groups and experimental pipeline. Male Yorkshire pigs $(\mathrm{n}=17)$ weighing $57.5 \pm 12 \mathrm{~kg}($ mean $\pm \mathrm{SD})$ were studied under normal $(n=6)$ and heart failure $(H F)$ model $(n=11)$ groups. For SG neural data collection, the animals were sedated with tiletamine and zolazepam (Telazol, 4-8mg/kg) 
intramuscularly, intubated, and maintained under general anesthesia with inhaled isoflurane (2\%). Continuous intravenous saline $(8-10 \mathrm{ml} / \mathrm{kg} / \mathrm{h})$ was infused throughout the protocol and animals were temperature maintained using heated water blankets $\left(37^{\circ} \mathrm{C}-38^{\circ} \mathrm{C}\right)$. Median sternotomy by an incision down the midline of the entire sternum (Fig. 1A) was performed to have a wide view of the thoracic region. The pericardium was opened to expose the heart and both stellate ganglia. After surgical procedures, animals were transitioned to alpha-chloralose anesthesia $(6.25 \mathrm{mg} / 125 \mathrm{~mL} ; 1 \mathrm{~mL} / \mathrm{kg}$ for bolus, $20-35 \mathrm{~mL} / \mathrm{kg}$ or titrated to effect for maintenance) with supplemental oxygen $(2 \mathrm{~L} /$ minute) for in vivo neural recordings from the left stellate ganglion (Fig. 1A). The left carotid artery was exposed, and a pressure catheter (SPR350, Millar Inc., Houston, TX) was inserted to continuously monitor left ventricular pressure (LVP). Additionally, three-lead surface electrocardiogram (ECG), respiratory pressure (RP) were monitored continuously, and sampled at $1 \mathrm{kHz}$. Arterial blood gas contents were monitored at least hourly to ensure appropriate experimental conditions. At the end of the protocol, animals were euthanized under deep sedation of isoflurane and cardiac fibrillation was induced.

Heart failure model was created with implanted pacemakers (Viva Cardiac Resynchronization Therapy-Pacemaker, Biotronik, Lake Oswego, OR), as previously described [27], visualized in Figure 1D. After implantation, animals had a recovery period of 48 hours and chronic bigeminy pacing was initiated from the right ventricle. This process produces premature ventricular contractions (PVCs) which lead to cardiomyopathy, also known as PIC-induced cardiomyopathy [28]. To confirm the progression of cardiomyopathy, echocardiography was performed, before and after implantation. After the animals have been confirmed to have cardiomyopathy (referred as $\mathrm{HF}$ animals) at eight weeks after implantation, surgical procedures described above were performed, and extracellular recordings obtained from the left stellate ganglion. It should be 
noted that a subset of HF animals $(n=6)$ underwent an intervention, epicardial application of resiniferatoxin (RTX) to study its effects on the progression of cardiomyopathy as a separate study. However no significant effect of RTX was noted in any of the echocardiographic, serum, physiological, and autonomic tests [27]. Hence, in this work, we combined RTX-treated HF animals with untreated HF animals.

\section{Stellate Ganglion Neural Recordings}

For each animal, a 16-channel, linear, single shank microelectrode array (LMA, Microprobes, Gaithersburg, MD) was inserted in the craniomedial pole of the left stellate ganglion (Fig. 1A). The LMA (Fig. 1B) consisted of a polyimide tube of $10 \mathrm{~mm}$ that contains recording sites, and a stainless steel tip of $1 \mathrm{~mm}$. Polyimide tube hosted a total of 16 platinum-iridium recording sites with $25 \mu \mathrm{m}$ radius, separated by $500 \mu \mathrm{m}$ intra-electrode spacing. A microelectrode amplifier (Model 3600, A-M Systems, Carlsborg, WA) was used to amplify (gain of 1000-2500) and filter $(300 \mathrm{~Hz}-3 \mathrm{kHz}$ band-pass filter) the acquired signals. The signals were transferred to a data acquisition platform (Power 1401, Cambridge Electronic Design, Cambridge, UK) and recorded using Spike2 software (Cambridge Electronic Design, Cambridge, UK). All data were processed in Python and MATLAB.

\section{Experimental Protocol}

We have shown in our previous work that spike rate increases within 90-minutes of electrode insertion, hence a stabilization time is required after the insertion takes place [26]. To accomplish this, all experiments included extended baseline recordings lasting approximately three hours. After baseline, the following stressors were applied in random order: inferior vena cava (IVC) occlusion to reduce cardiac preload, aortic occlusion (AOC) t increase cardiac afterload, decremental right ventricular (RV) pacing to induce electromechanical dyssynchrony (simulate a 
ventricular arrhythmia), respiratory stress to modulate respiration dynamics, mechanical touch of heart and elbow, and epicardial application of bradykinin $(20 \mu \mathrm{g} / \mathrm{ml})$ and capsaicin $(20 \mu \mathrm{g} / \mathrm{ml})$. Stressor information have been detailed in Supplementary Material 1.

\section{Signal Processing \& Time Series Analysis}

A high-level description of signal processing pipeline was provided in Fig. 2, each step was described in this section. Supporting codes were made available at GitHub.

\section{Unsupervised Spike Detection with Competitive Masking Algorithm}

Due to the unknown noise floor of the electrode array and the unknown neuron populations in SG, we followed an unsupervised approach for neural spike detection rather than conventional threshold-based approaches, which was detailed in prior work [26]. The algorithm initializes positive and negative barriers starting from the largest positive or negative signal amplitude and scans for crossings above and below the barriers iteratively. In each iteration, the detected spikes (signals crossing the barrier) are masked as a zero signal, and the barriers are further reduced in the next iteration. The barriers are brought closer to zero until a minimal number of crossings occur. This competitive masking enabled detection of smaller spikes that would otherwise not be possible with a single threshold-based algorithm.

\section{Cofluctuation \& Event Rate Definitions}

\section{i. Coactivity Matrix}

We developed a 16 x 16 correlation matrix ( 4 x 4 version shown in Fig. 3B, n=4) to investigate spatial coherence among neural populations in different regions of the SG spanned by 16 electrodes (Supplementary Fig. 1 shows a correlation matrix). The correlation matrix was constructed using pairwise Pearson's correlation of rolling spike rate windows. Note that the 
rolling mean of spike rate and the rolling standard deviation of spike rate are both considered and referred to as Spike Rate MEAN and Spike Rate $_{\mathrm{STD}}$ respectively in y-axis of Fig. 3A. For convenience these are referred to as 'spike rate' in what follows when both are implied. For instance, $R_{12}$ is the Pearson's correlation coefficient $(\mathrm{R})$ corresponding to channels 1 and 2 , shown as the red and blue windows respectively in Fig. 3A. In Fig. 3B, correlation matrix, there are $n-1=3$ superdiagonals and these are stacked vertically in Fig. 3Ci at each time point beginning with the first superdiagonal $\left(\mathrm{R}_{12}, \mathrm{R}_{23}, \mathrm{R}_{34}\right)$ at the bottom and subsequently referred to as the coactivity matrix. For this work, the superdiagonal of the 16-channel LMA's correlation matrix $(n=16)$ contains $\frac{\square *(\square-1)}{2}=120$ pairwise correlations at each time point, which resulted in 120 rows in the coactivity matrix. Vertical stacking of the superdiagonal pairwise correlations as a coactivity matrix helped us visualize channel-wise correlations running through the experiment, separated by multiples of the intra-electrode distance between channels $(500 \mu \mathrm{m})$.

\section{ii. Cofluctuations and Event Rate}

The cofluctuation time series was extracted from the coactivity matrix by calculating the percentage of channels exceeding a threshold, $C$, at each time point in the coactivity matrix $(\mathrm{R}>$ C, Fig. 3Cii). Regions in time where the cofluctuation time series exceeds a threshold $\mathrm{T}$ are termed as 'events' (Fig. 3Ciii). These events (Fig. 3Ciii) represent times wherein high cofluctuation is occurring across stellate channels both in space and time. These are converted to an event rate (ER) for each of the Spike Rate MEAN $_{\text {and Spike Rate }}$ STD over a duration in an experiment using:

$$
E R_{M E A N \text { or STD }}=\frac{\# \text { of MEAN or STD Events }}{\text { Duration }(s)}
$$


For clarity, $E R_{M E A N}$ implies that ER was calculated from cofluctuation time series computed from Spike Rate $\mathrm{MEAN}$, and $E R_{S T D}$ implies that ER was calculated from cofluctuation time series computed from Spike Rate $\mathrm{STD}$.

\section{iii. Cofluctuation Probability Distribution}

We observed that cofluctuation time series at each threshold (R, shown in Fig. 3Cii) mostly possess log-normal distribution, which indicates the natural logarithm of cofluctuation time series is normally distributed with mean $\mu$ and standard deviation $\sigma$ :

$$
\ln \left(\text { Cofluctuation }_{M E A N} \text { OR STD }\right) \sim N(\mu, \sigma)
$$

With probability density function (PDF):

$$
P D F_{\text {Cofluctuation }}(x)=\frac{1}{x \sigma \sqrt{2 \pi}} \exp \left(-\frac{(\ln x-\mu)^{2}}{2 \sigma^{2}}\right)
$$

The log-normal fits of cofluctuation time series (Fig. 7) were obtained using Python SciPy package, with statistics and random numbers module (scipy.stats) [29].

\section{iv. Bootstrapping and Selection of Optimal Thresholds}

Each event rate was calculated based on two thresholds, C and T. The first threshold (C, Fig. 3Cii) was used to obtain a percent series of channels exceeding a correlation threshold value placed on the coactivity matrix to obtain the cofluctuation series. The second threshold (T, Fig. 3Cii) was applied as a percent threshold on the cofluctuation series to extract events and an event rate for a specified duration. These thresholds were optimally chosen for each animal with respect to a cost function. We developed a bootstrapping procedure to select the optimal values for the two thresholds that yielded the narrowest confidence interval width of the bootstrapped event rate histograms. The optimization procedure is explained in Fig. 4A using a surrogate coactivity matrix R. Briefly, multiple cofluctuation time series were created with varying 
thresholds C (60-90\% with 15\% increments). Each were bootstrapped 1000 times and event rates at varying thresholds $\mathrm{T}$ were calculated from bootstrapped cofluctuation series. Multiple event thresholds T (40-90\% with $10 \%$ increments) were applied to ensure that the parameter space was covered. For each bootstrapped $(\mathrm{C}, \mathrm{T})$ pair, events and event rates were obtained (shown as red triangles in Fig. 4A, B). For each of the bootstrapped C, T pair, histograms of event rates were constructed to determine the $95 \%$ confidence intervals (CI, Fig. 4C). Individualized to each animal, optimum $\mathrm{C}$, T pairs were determined based on the narrowest $\mathrm{CI}$ that results in nonzero event rate (an optimum pair was shown in Fig. 4D that resulted in the narrowest CI with nonzero ER). Using this approach, the optimum $\mathrm{C}$, T pairs for each animal were listed and these pairs were used throughout related analyses (Fig. 4E).

\section{Neural Specificity \& Entropy Definition}

\section{i. $\quad$ Neural Specificity}

The neural specificity metric developed in our prior work [26] was used to compare the degree of stellate neural population phase-locking to LVP waveform in both animal groups, as a measure of cardiac control performance metric, as summarized in Fig. 5. Briefly, the metric was computed by subtracting a normalized histogram of LVP (i.e., functional target) computed at randomized spike times over many cycles from the histogram of LVP computed at spike times during those cycles for multiple windows for the course of the experiment. To explain the construction of the metric with LVP as an example, a representative window is shown in Fig. 5A with the spikes shown as green dots over LVP waveform. The following steps outline the construction of the metric for the representative LVP window: 
1. Following the notation used in [26], the normalized sliding window histogram of $L V P_{j}$ at a time $t_{j}$ with $1 \leq j \leq N_{\max }$ over $M$ bins was defined for the $k^{\text {th }}$ bin as:

$$
H\left(L V P_{j}\right)_{k}=\frac{h\left(L V P_{j}\right)_{k}}{\sum_{k=1}^{k=M} h\left(L V P_{j}\right)_{k}}
$$

2. First, the LVP value at the spike time $t_{j}$ in the window shown was computed, $S L V P_{j}$, followed by its normalized histogram $H\left(S L V P_{j}\right)_{k}$ for the $k^{\text {th }}$ bin (green line plot, Fig. 5B). The normalized histogram for the $k^{t h}$ bin is computed again for the LVP values with spike times randomized to obtain $H\left(L V P_{j}\right)_{k}$ (pink line plot, Fig. 5B).

3. The neural specificity, $A_{j k}$, for the $k^{t h}$ bin was calculated using:

$$
A_{j k}=H\left(S L V P_{j}\right)_{k}-H\left(L V P_{j}\right)_{k}
$$

with $A_{j k}$ mapped to $[-1,0,1]$ using a mapping threshold $(0.5)$. (The subtracted histogram with applied mapping threshold was shown in Fig. 5C).

From the $k^{\text {th }}$ bin passed through the mapping threshold, we obtain the following mappings:

i. $A_{j k}=1$, shown in yellow representing greater bias towards $L V P_{j}$ value than that due to random sampling.

ii. $A_{j k}=-1$, shown in purple representing lesser bias towards $L V P_{j}$ value than that due to random sampling.

iii. $A_{j k} \approx 0$, shown in teal representing near-zero bias towards $L V P_{j}$ value than that due to random sampling.

The neural specificity metric for the entire experiment was constructed by repeating steps two and three with mapping threshold applied for all sliding windows in the experiment (Fig. 5D). 


\section{ii. Entropy}

The Shannon's entropy of each window in the neural specificity metric was computed at each time point following its construction.

The entropy of the neural specificity was computed at each timepoint $t_{i}$ based on the first difference of the neural specificity $A_{t_{i}}$, i.e., $\Delta A_{t_{i}}$ (Fig. 5D). The magnitude of the change in neural specificity (-1, 01 ; purple, teal, yellow) for the timepoint $t_{i}$ has the following values:

1. $\Delta A_{t_{i}}=0$ represents no change in neural specificity

2. $\Delta A_{t_{i}}=1$ represents a change in neural specificity of magnitude 1

3. $\Delta A_{t_{i}}=2$ represents a change in neural specificity of magnitude 2

The entropy (E) follows as:

$$
E=-\sum_{\Delta A_{t_{i}}=0}^{\Delta A_{t_{i}}=2} p\left(\Delta A_{t_{i}}\right) * \log _{3}\left(p\left(\Delta A_{t_{i}}\right)\right)
$$

The base of the entropy was chosen as 3 for the 3 values present in the first difference computation $(0,1,2)$. The entropy time series was obtained by calculating the entropy for all time points in the neural specificity metric (with the entropy calculation of the representative time point shown as a red dot in Fig. 5E). Note that $\mathrm{E}$ was computed for each of mean $\left(\right.$ ENTROPY $\left._{\text {MEAN }}\right)$ and standard deviation $\left(\right.$ ENTROPY $\left._{\text {STD }}\right)$ of entropy time series.

\section{iii. Event Entropy}

In order to study cofluctuations in stellate neural activity in terms of function in closed-loop control, we considered the entropy time series inside and outside the event regions found from cofluctuation separately. The mean and standard deviation of extracted entropy values of each 
channel of the LMA probe during events and non-events were computed for each experiment, referred to as "event entropy".

\section{Dataset and Statistical Analysis}

Statistical analyses were performed in MATLAB Statistics \& Machine Learning Toolbox (version R2021a) and Python SciPy Library (version 3.8.5).

\section{Outcome Measures}

Within the signal processing pipeline described in Fig. 3, the event rate measures, $E R_{M E A N}$ and $E R_{S T D}$, were used to summarize the cofluctuation time series for each animal. For the entropy calculation each channel had a mean and standard deviation of entropy time series, resulting in 32 measures of entropy per animal (16 ENTROPY MEAN numbers per animal, 16 ENTROPY $_{\text {STD }}$ numbers per animal, corresponding to each channel).

\section{Sample Size Breakdown}

Two channels were excluded from two animals due to insufficient signal quality. Within baseline event rate analyses, all animals had sufficient neural data ( $\mathrm{n}=17$ animals, 6 normal, $11 \mathrm{HF})$. Entropy analyses for three heart failure animals were excluded due to insufficient LVP quality resulting in $\mathrm{n}=14$ animals ( 6 normal, $8 \mathrm{HF}$ ). The stressors of one normal animal and three $\mathrm{HF}$ animals led to ventricular fibrillation during the protocol and no usable stressor data were available leaving $\mathrm{n}=10$ animals ( 5 normal, $5 \mathrm{HF}$ ) for analyses.

\section{Statistical Analysis}

We conducted analogous analyses during baseline and stress states. The computed metrics during stress were normalized to corresponding baselines either by division (i.e., ER MEAN,STRESS / 
ER $_{\text {MEAN, BASELINE) or subtraction (i.e., ER }}$ MEAN,STRESS - ER MEAN,BASELINE) and used in statistical analyses.

Shapiro-Wilk test was used to determine normality. For variables that result in a single number per animal (such as ER MEAN, BASELINE $_{\text {and }} \mathrm{ER}_{\mathrm{STD}}$, BASELINE, Fig. 6A-B), independent samples ttests or Wilcoxon rank-sum tests were used for normal or non-normal data, respectively, to understand differences between animal groups. For variables that have multiple numbers per

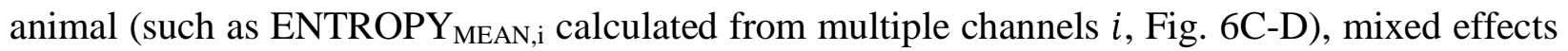
models were constructed in MATLAB Statistics and Machine Learning Toolbox [30, 31] with the model details explained below.

For baseline and stress entropy investigations (Fig. 6C-D, Fig. 8C-D), the computed and/or normalized metric (i.e., ENTROPY MEAN, BASELINE or ENTROPY MEAN, (STRESS/BASELINE) was assigned as the outcome variable; animal type (normal / HF) was assigned as fixed effect; and channel number (1-16) and animal ID were assigned as random effects. For event entropy (i.e., regional entropy with relation to events and non-events), models were constructed such as the event / non-event entropy is the outcome variable; event type (event / non-event), animal type (normal / HF), and coactivity computation type (mean / std) are fixed effects; and channel number, animal ID, and baseline entropy (type matching the outcome entropy's type, mean or std) are the random effects.

For all analyses using mixed effects, the $\beta$ coefficients (fixed effects estimates), p-values, effect sizes $\left(d_{R M}\right.$, based on repeated measures Cohen's $\left.d_{R M}[32]\right), 95 \%$ confidence intervals (CI) of $\beta$ coefficients (lower, upper) were reported in results in $\left(\beta, \pm C I, d_{R M}, p\right)$ format. $\beta$ coefficients indicate the adjusted differences (units matching the outcome variable's unit) in one group compared to the other. For analyses with independent samples, p-values and independent 
samples effect sizes (d, based on Cohen's d) were reported in $(p, d)$ format. For all analyses, a two-sided $\mathrm{p} \square<\square 0.05$ denoted statistical significance.

\section{Results}

In all studies, an LMA probe was inserted to the left SG of each animal (Fig. 1A-B). A total of 16 channels of neural activity was collected along with simultaneous left ventricular pressure (LVP) for approximately six hours of continuous recordings per animal. Representative neural activity recording for a single channel, LVP, and representative spike trains were displayed for normal and heart failure animals in Fig. 1A. A total of 17 Yorkshires (6 normal, 11 heart failure, Fig. 1D) underwent the terminal experiment described in Fig. 1E. Upon the signal processing pipeline described in Fig. 2, we computed two event rate measures per animal as the final product representing the cofluctuations $\left(\mathrm{ER}_{\mathrm{MEAN}}, \mathrm{ER}_{\mathrm{STD}}\right)$. As the metric representing the neural

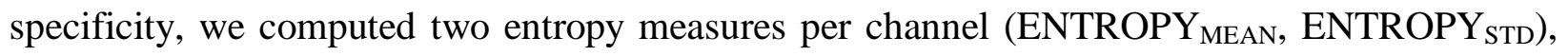
resulting in a total of 16 ENTROPY $_{\text {MEAN }}$ and 16 ENTROPY $_{\text {STD per animal. Finally, we used }}$ these metrics to quantify: i) neural population dynamics (i.e., event rate measures), ii) neural specificity to target LVP, or cardiac control (i.e., entropy measures), and iii) Linkage between neural population dynamics and specificity (i.e., event entropy).

\section{Stellate Ganglion in Heart Failure Exhibits High Baseline Event Rate}

Fig. 6A-B show event rate outcomes grouped by heart failure (HF) and normal models. In baseline, HF animals show significantly higher event rates compared to normal animals for both $\operatorname{ER}_{\text {MEAN }}(p=0.011$, effect size $d=1.59)$ and $\operatorname{ER}_{\mathrm{STD}}(\mathrm{p}=0.023, \mathrm{~d}=1.48)$. The event time series for each animal is depicted in Supplementary Fig. 2. Within the event time series are 'events' or 
narrow regions in time where high cofluctuations are shown as level 1 . In addition, Supplementary Fig. 3 shows the cofluctuations for each animal, out of which the events time series were computed. We observe that the cofluctuations are more localized in HF animals with more heterogeneity.

\section{No Significant Difference in Event Rate during Stressors}

We also compared the change in event rate measures from baseline to stressors, normalized to baseline, computed from both ratio (ER stress $\left./ \mathrm{ER}_{\text {baseline}}\right)$ and absolute change $\left(\mathrm{ER}_{\text {stress }}-\mathrm{ER}_{\text {baseline }}\right)$ for each animal. In contrast to the significant event rate differences between animal groups in baseline, there was no statistically significant change in stress responses pertaining event rate. In

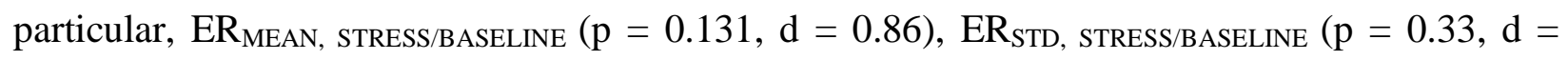
0.53), ER MEAN, STRESS-BASELINE $(\mathrm{p}=0.099, \mathrm{~d}=0.90)$, ER $_{\mathrm{STD}}$, STRESS-BASELINE $(\mathrm{p}=0.444, \mathrm{~d}=0.43)$ did not yield statistically significant results between animal groups.

\section{Heart Failure Animals Have Heavy Tailed Cofluctuation Distributions}

We explored the statistical distribution of the cofluctuation time series. We observed that cofluctuation time series at optimal thresholds mostly possess log-normal distribution in normal animals. Fig. 7 shows log-normal fits for each animal group for Cofluctuation MEAN and Cofluctuation $_{\mathrm{STD}}$ time series, along with $68 \%$ confidence interval (CI) bounds, mean of fit $\left(\mu_{\mathrm{FIT}}\right)$ and standard deviation of fit $\left(\sigma_{\text {FIT }}\right)$. Normal animals (Fig. 7A) exhibit narrow confidence intervals, lower $\mu_{\text {FIT }}$ and $\sigma_{\text {FIT }}$ values, and tighter log-normal fits. In contrast, HF animals (Fig. 
7B) exhibit wider confidence intervals, higher $\mu_{\text {FIT }}$ and $\sigma_{\text {FIT }}$ values, and looser log-normal fits. Of note, HFanimals have heavy tails ranging further outside of confidence bounds.

Stellate Ganglion in Heart Failure Shows Greater Variation in Neural Specificity to LVP in Baseline

We next examined the neural specificity to LVP in baseline, quantified by entropy measures. Fig. 6C-D shows ENTROPY MEAN $_{\text {and ENTROPY }}$ in in baseline, grouped by animals. Compared to normal group, stellate ganglion of $\mathrm{HF}$ group animals exhibited significantly higher ENTROPY $_{\text {STD }}$ (variation in entropy) in baseline (Fig. 6D, adjusted $\beta=0.01$ n.u., $95 \% \mathrm{CI}= \pm$ 0.01 n.u., $\left.d_{\text {RM }}=0.73, p=0.009\right)$. However, there is no significant difference in ENTROPY MEAN (mean entropy) between animal groups. (Fig. $6 \mathrm{C}, \beta=0.04$ n.u., \pm 0.05 n.u., $\mathrm{d}_{\mathrm{RM}}=0.82, \mathrm{p}=$ 0.087).

Saturated Neural Specificity during Stress in Heart Failure

To quantify the degree of cardiac control during stress, we examined the changes in neural specificity as quantified by entropy measures in all animals, normalized to their baseline,

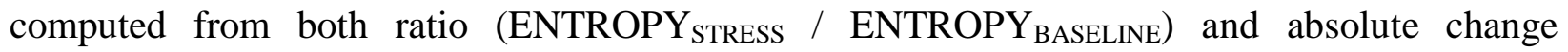

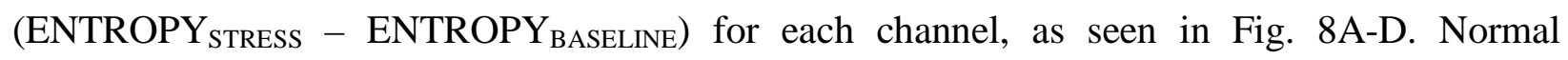

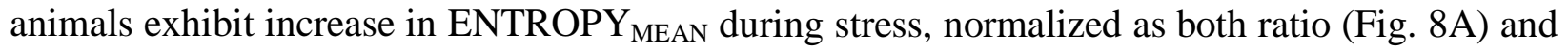
absolute change from baseline (Fig. 8B). Interestingly, HF group animals show comparatively less change from their baseline for ENTROPY ${ }_{\text {MEAN }}$, indicating saturated cardiac control of LVP during stressors. In particular, compared to normal animals, HF animals exhibit significantly 
lower ENTROPY MEAN, STRESS/BASELINE (Fig. 8A, $\beta=-0.25$ n.u., \pm 0.23 n.u., $d_{\text {RM }}=0.56, p=0.035$ )

and ENTROPY MEAN, STRESS-BASELINE (Fig. 8B, $\beta=-0.08$ n.u., \pm 0.07 n.u., $\mathrm{d}_{\mathrm{RM}}=0.68, \mathrm{p}=0.021$ )

responses. For the variation in entropy, we observe that both groups exhibit decrease in variation in entropy relative to their baseline for both ratio and absolute changes, ENTROPY $\mathrm{STD}_{\text {, }}$

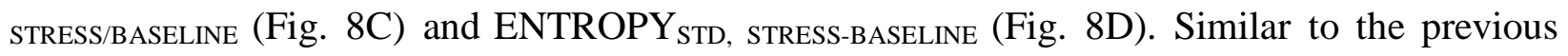
case, the variability in entropy during stress appears significantly lower in HF animals compared

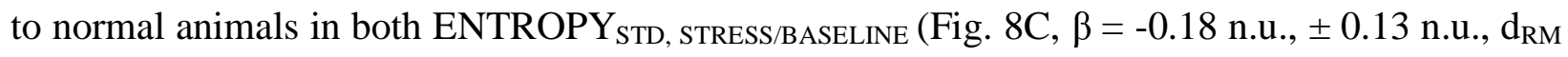

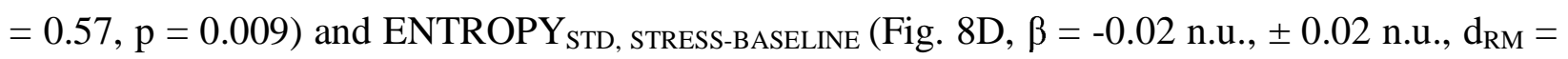
$0.97, \mathrm{p}=0.041)$

Neural Network Activity and Cardiac Control Linkage Depends on Animal Group and Cofluctuation Magnitude

Finally, we sought to explore the nature of cardiac control inside and outside short duration regions of high cofluctuation, i.e. 'events', characterized by strongly coherent stellate activity patterns. The relation between cardiac control and neural activity is represented here by using entropy to represent control and considering how control differs inside and outside events. The nature of the evolution of entropy during these events is termed 'event entropy'. First, we studied whether entropy changes inside and outside of events (Fig. 9A, C). Second, we studied whether entropy is sensitive to the animal group (Fig. 9B, D). Regardless of the animal group,

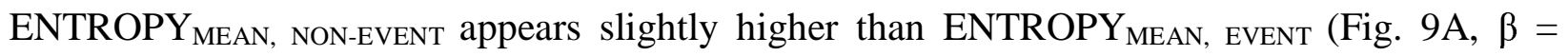
0.007 n.u., \pm 0.004 n.u., $\left.\mathrm{d}_{\mathrm{RM}}=0.07, \mathrm{p}<0.001\right)$. Similarly, ENTROPY STD, NON-EVENT appears

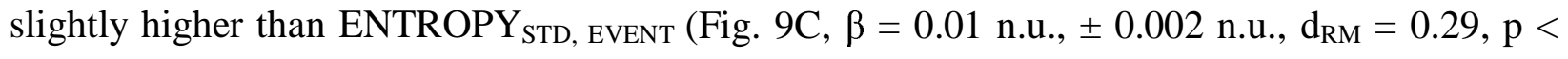
0.001). When we examined the contribution of animal group, we found no significant difference 


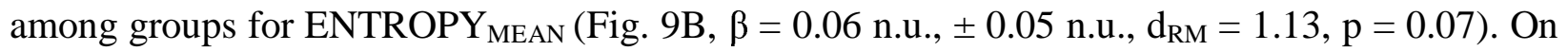
the other hand, HF animals exhibit significantly higher ENTROPY STD $_{\text {compared to normal }}$ animals (Fig. 9D, $\beta=0.02$ n.u., \pm 0.02 n.u., $d_{R M}=0.75, p=0.012$ ). These analyses point out the fact that linkage between neural network function and cardiac control differs inside and outside of events and between animal groups in the stellate ganglion.

\section{Discussion}

In this work, we performed a novel investigation of stellate ganglion neural population dynamics and neural specificity to continuous left ventricular blood pressure in healthy and heart failure Yorkshire models. The present findings expand on our prior investigations in healthy animals, which laid the groundwork for quantifying neural specificity to cardiac cycles [44]. In the current work, we investigated neural networking and cardiopulmonary integration differences in healthy and pathological states.

Prevalence of Short-Lived Baseline Cofluctuations in Stellate Ganglion Activity during Heart

\section{Failure}

Our findings indicate that SG neural populations in heart failure models exhibit short-lived cofluctuations which are translated into events. In contrast, cofluctuation patterns in normal stellate ganglion appears to be more randomand of generally lower amplitude, resulting in fewer events (Supplementary Fig. 2 and 3). The heavy tail feature is only apparent in the heart failure model and therefore may be linked to an expression of cardiac pathology by introducing outlier events. Log-normal, skewed distributions are frequently observed in biological systems as they possess emergent and collective properties as a result of complex interactions [33]. It should be 
noted that the choice of fit distribution (log-normal) was empirical. We investigated a power law distribution (associated with heavy tails [34]) which was only able to capture the heavy tails leaving the log-normal as the preferred alternative.

\section{Baseline Cardiac Control Exhibits Greater Unpredictability During Heart Failure}

In prior work, we identified neural spiking specificity toward near-peak systole of LVP waveform in healthy animals [26]. With the current results comparing normal versus HF animals, this metric serves as a control network performance indicator. Our results show that baseline cardiac control network performance changes from healthy to diseased states, with greater variation in entropy (i.e., unpredictability) for heart failure animals compared to normal animals. This finding may extend to other pathologies for which the cardiac control hierarchy is disrupted.

\section{Limited Sympathetic Reserve: Saturated Cardiac Control during Stress in Heart Failure}

Our findings on changes in entropy between baseline and stressed states are noteworthy (Fig. 8A-D). While heart failure animals show greater variation in unpredictability during baseline

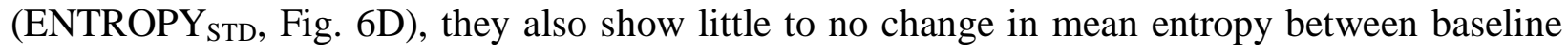
and stress (ENTROPY $Y_{\text {MEAN }}$, Fig. 8A-B). This finding is consistent with studies demonstrating increased baseline sympathetic activity in the setting of HF [35-38] which may imply a limited capacity to further increase sympathetic activity. This limited sympathetic reserve is also observed in other disease domains, such as saturated physiological response to traumatic reminders in posttraumatic stress disorder [39]. On the other hand, both HF and normal animals 
show decreased variation in entropy during stressed states. HF animals show a greater decrease in variation than normal animals consistent with a limited sympathetic reserve.

\section{Neural Network Activity is Linked to Cardiac Control}

We linked neural network functioning to cardiac control via an event entropy metric. Based on the effect size $\left(\mathrm{d}_{\mathrm{RM}}\right)$, event entropy magnitude appears to be higher with greater variation in $\mathrm{HF}$ animals compared to normal animals (Fig. B-D). This implies a level of increased difficulty in cardiac control for animals in heart failure over normal animals. It should be noted that the effect sizes for event versus non-event comparisons were small to medium, which potentially indicates a larger study is necessary to better understand the physiological contributions from event type.

\section{Limitations}

Of note, we did not observe significance in event rates during stressors, despite high effect sizes. We believe that this may be attributed to the short duration of the stressors, and the current experimental protocol may not resolve the high cofluctuation dynamics during stress. A potential solution is extending the duration of stressors by keeping the stressor amplitude lower to monitor long-term changes in these metrics under stress relevant to the cardiac pathology (e.g., low-level pacing protocols). Measurement of these neurocardiac metrics during slow, quasi-static application of clinically-relevant stressors should provide unique opportunities to investigate unresolved questions in this area. A similar design has been developed in anesthesiology research $[40,41]$ to uncover important features of integrative networks in the brain that were otherwise unavailable. 
Another limitation of the study lies in the absence of multiple-class pathologies (i.e., heart failure models and other reproducible models) and in the absence of stratified pathologies (i.e., animal models with varying degrees of heart failure). Future studies should focus on expanding the dataset to examine how these metrics change with varying pathologies or varying disease models.

\section{Conclusion}

Our findings are aligned with a growing consensus in neuroscience that changes in neural activity through space and time are associated with the onset and development of specific pathologies. Neuroscience studies in different domains have been undertaken to understand the underpinnings of pathologies and information processing. For instance, spatiotemporal brainwide fluctuations were reported to reveal major depression vulnerability [42]. A different study reported that brain's functional connectivity is driven by high-amplitude cofluctuations in baseline states, and these fluctuations encode subject-specific information during experimental tasks [43]. Furthermore, neural specificity phenomenon was observed within the context of visual neural circuits with the difference that neural activity time-locks to visual stimuli [44]. In our work, the primary observation has been that event-based processing within the stellate ganglion and its relationship to cardiac control is strongly sensitive to disruptions of the cardiac milieu. Understanding the evolution of networked stellate ganglion processing inside and outside these high cofluctuation time domains during baseline and stressed states will provide insight into unresolved conditions such as sudden cardiac death. 


\section{References}

[1] J. L. Ardell et al., "Translational neurocardiology: preclinical models and cardioneural integrative aspects," The Journal of Physiology, https://doi.org/10.1113/JP271869 vol. 594, no. 14, pp. 38773909, 2016/07/15 2016, doi: https://doi.org/10.1113/JP271869.

[2] W. Deyell Marc, D. Krahn Andrew, and J. Goldberger Jeffrey, "Sudden Cardiac Death Risk Stratification," Circulation Research, vol. 116, no. 12, pp. 1907-1918, 2015/06/05 2015, doi: 10.1161/CIRCRESAHA.116.304493.

[3] G. Kember, J. A. Armour, and M. Zamir, "Neural control hierarchy of the heart has not evolved to deal with myocardial ischemia," Physiological Genomics, vol. 45, no. 15, pp. 638-644, 2013/08/01 2013, doi: 10.1152/physiolgenomics.00027.2013.

[4] O. A. Ajijola et al., "Focal myocardial infarction induces global remodeling of cardiac sympathetic innervation: neural remodeling in a spatial context," American Journal of Physiology-Heart and Circulatory Physiology, vol. 305, no. 7, pp. H1031-H1040, 2013/10/01 2013, doi: 10.1152/ajpheart.00434.2013.

[5] S. Han et al., "Electroanatomic Remodeling of the Left Stellate Ganglion After Myocardial Infarction," Journal of the American College of Cardiology, vol. 59, no. 10, pp. 954-961, 2012/03/06/ 2012, doi: https://doi.org/10.1016/j.jacc.2011.11.030.

[6] O. A. Ajijola et al., "Remodeling of stellate ganglion neurons after spatially targeted myocardial infarction: Neuropeptide and morphologic changes," Heart Rhythm, vol. 12, no. 5, pp. 10271035, 2015/05/01/ 2015, doi: https://doi.org/10.1016/j.hrthm.2015.01.045.

[7] K. Nakamura, O. A. Ajijola, E. Aliotta, J. A. Armour, J. L. Ardell, and K. Shivkumar, "Pathological effects of chronic myocardial infarction on peripheral neurons mediating cardiac neurotransmission," Autonomic Neuroscience, vol. 197, pp. 34-40, 2016/05/01/ 2016, doi: https://doi.org/10.1016/j.autneu.2016.05.001.

[8] O. A. Ajijola et al., "Coronary Sinus Neuropeptide Y Levels and Adverse Outcomes in Patients With Stable Chronic Heart Failure," JAMA Cardiology, vol. 5, no. 3, pp. 318-325, 2020, doi: 10.1001/jamacardio.2019.4717.

[9] O. A. Ajijola et al., "Extracardiac Neural Remodeling in Humans With Cardiomyopathy," Circulation: Arrhythmia and Electrophysiology, vol. 5, no. 5, pp. 1010-1116, 2012/10/01 2012, doi: 10.1161/CIRCEP.112.972836.

[10] M. Vaseghi et al., "Sympathetic innervation of the anterior left ventricular wall by the right and left stellate ganglia," Heart Rhythm, vol. 9, no. 8, pp. 1303-1309, 2012/08/01/ 2012, doi: https://doi.org/10.1016/j.hrthm.2012.03.052.

[11] M. Vaseghi et al., "Cardiac Sympathetic Denervation for Refractory Ventricular Arrhythmias," Journal of the American College of Cardiology, vol. 69, no. 25, pp. 3070-3080, 2017/06/27/ 2017, doi: https://doi.org/10.1016/j.jacc.2017.04.035. 
[12] O. A. Ajijola et al., "Bilateral Cardiac Sympathetic Denervation for the Management of Electrical Storm," Journal of the American College of Cardiology, vol. 59, no. 1, pp. 91-92, 2012/01/03/ 2012, doi: https://doi.org/10.1016/j.jacc.2011.09.043.

[13] W. Wang and I. H. Zucker, "Cardiac sympathetic afferent reflex in dogs with congestive heart failure," American Journal of Physiology-Regulatory, Integrative and Comparative Physiology, vol. 271, no. 3, pp. R751-R756, 1996/09/01 1996, doi: 10.1152/ajpregu.1996.271.3.R751.

[14] R. Ma, I. H. Zucker, and W. Wang, "Central gain of the cardiac sympathetic afferent reflex in dogs with heart failure," American Journal of Physiology-Heart and Circulatory Physiology, vol. 273, no. 6, pp. H2664-H2671, 1997/12/01 1997, doi: 10.1152/ajpheart.1997.273.6.H2664.

[15] W. W. Chen, X. Q. Xiong, Q. Chen, Y. H. Li, Y. M. Kang, and G. Q. Zhu, "Cardiac sympathetic afferent reflex and its implications for sympathetic activation in chronic heart failure and hypertension," Acta Physiologica, https://doi.org/10.1111/apha.12447 vol. 213, no. 4, pp. 778794, 2015/04/01 2015, doi: https://doi.org/10.1111/apha.12447.

[16] H.-J. Wang, G. J. Rozanski, and I. H. Zucker, "Cardiac sympathetic afferent reflex control of cardiac function in normal and chronic heart failure states," The Journal of Physiology, https://doi.org/10.1113/JP273764 vol. 595, no. 8, pp. 2519-2534, 2017/04/15 2017, doi: https://doi.org/10.1113/JP273764.

[17] W.-Z. Wang, L. Gao, H.-J. Wang, I. H. Zucker, and W. Wang, "Interaction between cardiac sympathetic afferent reflex and chemoreflex is mediated by the NTS AT1 receptors in heart failure," American Journal of Physiology-Heart and Circulatory Physiology, vol. 295, no. 3, pp. H1216-H1226, 2008/09/01 2008, doi: 10.1152/ajpheart.00557.2008.

[18] H.-J. Wang, W. Wang, K. G. Cornish, G. J. Rozanski, and I. H. Zucker, "Cardiac Sympathetic Afferent Denervation Attenuates Cardiac Remodeling and Improves Cardiovascular Dysfunction in Rats With Heart Failure," Hypertension, vol. 64, no. 4, pp. 745-755, 2014/10/01 2014, doi: 10.1161/HYPERTENSIONAHA.114.03699.

[19] L. Gao, H. D. Schultz, K. P. Patel, I. H. Zucker, and W. Wang, "Augmented Input From Cardiac Sympathetic Afferents Inhibits Baroreflex in Rats With Heart Failure," Hypertension, vol. 45, no. 6, pp. 1173-1181, 2005/06/01 2005, doi: 10.1161/01.HYP.0000168056.66981.c2.

[20] L. Gao et al., "Cardiac sympathetic afferent stimulation augments the arterial chemoreceptor reflex in anesthetized rats," Journal of Applied Physiology, vol. 102, no. 1, pp. 37-43, 2007/01/01 2007, doi: 10.1152/japplphysiol.00681.2006.

[21] J. A. Armour, "Synaptic transmission in the chronically decentralized middle cervical and stellate ganglia of the dog," Canadian Journal of Physiology and Pharmacology, vol. 61, no. 10, pp. 1149-1155, 1983/10/01 1983, doi: 10.1139/y83-171.

[22] J. A. Armour, "Activity of in situ stellate ganglion neurons of dogs recorded extracellularly," Canadian Journal of Physiology and Pharmacology, vol. 64, no. 2, pp. 101-111, 1986/02/01 1986, doi: 10.1139/y86-016.

[23] J. A. Armour, K. Collier, G. Kember, and J. L. Ardell, "Differential selectivity of cardiac neurons in separate intrathoracic autonomic ganglia," American Journal of Physiology-Regulatory, Integrative and Comparative Physiology, vol. 274, no. 4, pp. R939-R949, 1998/04/01 1998, doi: 10.1152/ajpregu.1998.274.4.R939.

[24] K. Yoshie et al., "Cardiac TRPV1 afferent signaling promotes arrhythmogenic ventricular remodeling after myocardial infarction," JCI Insight, vol. 5, no. 3, 02/13/ 2020, doi: 10.1172/jci.insight. 124477.

[25] K. Yoshie et al., "Cardiac vanilloid receptor-1 afferent depletion enhances stellate ganglion neuronal activity and efferent sympathetic response to cardiac stress," American Journal of Physiology-Heart and Circulatory Physiology, vol. 314, no. 5, pp. H954-H966, 2018/05/01 2018, doi: 10.1152/ajpheart.00593.2017.

[26] K. B. Sudarshan et al., "A Novel Metric Linking Stellate Ganglion Neuronal Population Dynamics to Cardiopulmonary Physiology," American Journal of Physiology-Heart and Circulatory Physiology, 2021, doi: https://doi.org/10.1152/ajpheart.00138.2021. 
[27] Y. Hori et al., "Cardiac afferent signaling partially underlies premature ventricular contractioninduced cardiomyopathy," Heart Rhythm, vol. 18, no. 9, pp. 1586-1595, 2021/09/01/ 2021, doi: https://doi.org/10.1016/j.hrthm.2021.04.004.

[28] M. Sadron Blaye-Felice et al., "Premature ventricular contraction-induced cardiomyopathy: Related clinical and electrophysiologic parameters," Heart Rhythm, vol. 13, no. 1, pp. 103-110, 2016/01/01/ 2016, doi: https://doi.org/10.1016/j.hrthm.2015.08.025.

[29] P. Virtanen et al., "SciPy 1.0: fundamental algorithms for scientific computing in Python," Nature Methods, vol. 17, no. 3, pp. 261-272, 2020/03/01 2020, doi: 10.1038/s41592-019-0686-2.

[30] J. C. Pinheiro and D. M. Bates, "Unconstrained parametrizations for variance-covariance matrices," Statistics and Computing, vol. 6, no. 3, pp. 289-296, 1996/09/01 1996, doi: 10.1007/BF00140873.

[31] MathWorks. "Fit linear mixed-effects model documentation." MathWorks. https://www.mathworks.com/help/stats/fitlme.html (accessed September, 2021).

[32] D. Lakens, "Calculating and reporting effect sizes to facilitate cumulative science: a practical primer for t-tests and ANOVAs," (in English), Frontiers in Psychology, Review vol. 4, no. 863, 2013-November-26 2013, doi: 10.3389/fpsyg.2013.00863.

[33] G. Buzsáki and K. Mizuseki, "The log-dynamic brain: how skewed distributions affect network operations," Nature Reviews Neuroscience, vol. 15, no. 4, pp. 264-278, 2014/04/01 2014, doi: 10.1038/nrn3687.

[34] Y. S. Pavlova, D. Paez-Espino, A. Y. Morozov, and I. S. Belalov, "Searching for fat tails in CRISPR-Cas systems: Data analysis and mathematical modeling," PLOS Computational Biology, vol. 17, no. 3, p. e1008841, 2021, doi: 10.1371/journal.pcbi.1008841.

[35] R. M. Oren, P. J. Roach, H. P. Schobel, W. J. Berg, and D. W. Ferguson, "Sympathetic responses of patients with congestive heart failure to cold pressor stimulus," The American Journal of Cardiology, vol. 67, no. 11, pp. 993-1001, 1991/05/01/ 1991, doi: https://doi.org/10.1016/00029149(91)90173-I.

[36] W. N. Leimbach, B. G. Wallin, R. G. Victor, P. E. Aylward, G. Sundlöf, and A. L. Mark, "Direct evidence from intraneural recordings for increased central sympathetic outflow in patients with heart failure," Circulation, vol. 73, no. 5, pp. 913-919, 1986/05/01 1986, doi: 10.1161/01.CIR.73.5.913.

[37] T. S. Rector, M. T. Olivari, T. Barry Levine, G. S. Francis, and J. N. Cohn, "Predicting survival for an individual with congestive heart failure using the plasma norepinephrine concentration," American Heart Journal, vol. 114, no. 1, Part 1, pp. 148-152, 1987/07/01/ 1987, doi: https://doi.org/10.1016/0002-8703(87)90318-8.

[38] W. Ferguson David, J. Berg William, S. Sanders Jeffrey, and S. Kempf Joan, "Clinical and hemodynamic correlates of sympathetic nerve activity in normal humans and patients with heart failure: Evidence from direct micronenrographic recordings," Journal of the American College of Cardiology, vol. 16, no. 5, pp. 1125-1134, 1990/11/01 1990, doi: 10.1016/0735-1097(90)90544Y.

[39] S. P. Orr, L. J. Metzger, and R. K. Pitman, "Psychophysiology of post-traumatic stress disorder," Psychiatric Clinics of North America, vol. 25, no. 2, pp. 271-293, 2002/06/01/ 2002, doi: https://doi.org/10.1016/S0193-953X(01)00007-7.

[40] O. Akeju and E. N. Brown, "Neural oscillations demonstrate that general anesthesia and sedative states are neurophysiologically distinct from sleep," Current Opinion in Neurobiology, vol. 44, pp. 178-185, 2017/06/01/ 2017, doi: https://doi.org/10.1016/j.conb.2017.04.011.

[41] S. Chamadia et al., "Delta oscillations phase limit neural activity during sevoflurane anesthesia," Communications Biology, vol. 2, no. 1, p. 415, 2019/11/15 2019, doi: 10.1038/s42003-019-06643.

[42] R. Hultman et al., "Brain-wide Electrical Spatiotemporal Dynamics Encode Depression Vulnerability," Cell, vol. 173, no. 1, pp. 166-180.e14, 2018/03/22/ 2018, doi: https://doi.org/10.1016/j.cell.2018.02.012. 
[43] F. Zamani Esfahlani et al., "High-amplitude cofluctuations in cortical activity drive functional connectivity," Proceedings of the National Academy of Sciences, vol. 117, no. 45, p. 28393, 2020, doi: 10.1073/pnas.2005531117.

[44] J.-e. K. Miller, I. Ayzenshtat, L. Carrillo-Reid, and R. Yuste, "Visual stimuli recruit intrinsically generated cortical ensembles," Proceedings of the National Academy of Sciences, vol. 111, no. 38, p. E4053, 2014, doi: 10.1073/pnas.1406077111.

Figures and Legends

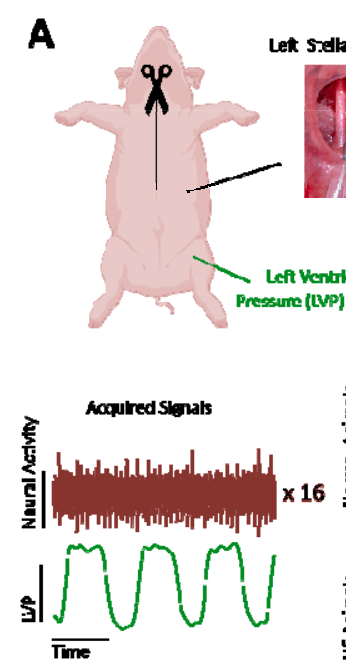

E Terminal Experiment
B
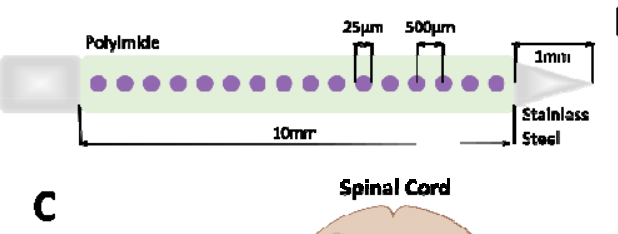

C
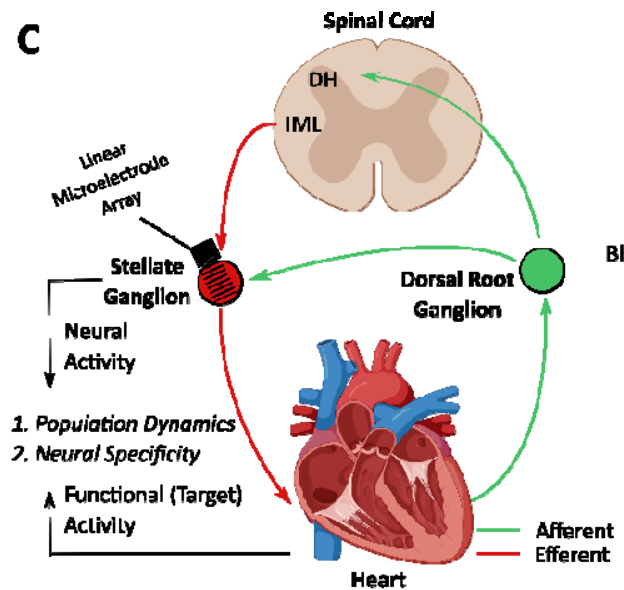

\begin{tabular}{|l|}
\hline ReJresentative Sflbe Tralns \\
\hline||||||||||||||||||||||||||||||||||$|~||||||||| \mid$
\end{tabular}

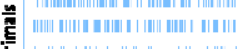

|

ह
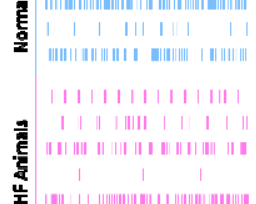

Tirne

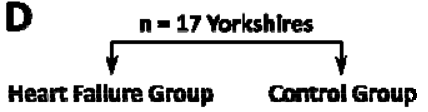

Heart Fallure Group control Grou

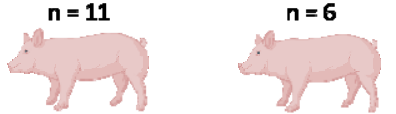

$\downarrow$

Pacemaker

Implantation

$\downarrow 48$ hours

Bigeminy Pacing On

$\downarrow 8$ weeks

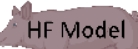

d)

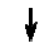

Termiral

Experiment

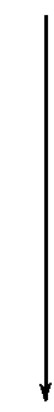

Terminal Experiment

Sedation

$\rightarrow$ Intubation

$\rightarrow$ Median Stemotomy

LMA nsertion

Extended Baseline Recordings

Stressors

Figure 1. Experimental workflow and overall concept.

A) A linear microelectrode array (LMA) was inserted to the left stellate ganglion (SG) for each animal. 16 channels of neural activity were collected along with simultaneous left ventricular pressure (LVP). Representative spike trains were displayed for each animal group.

B) Specifications of the LMA.

C) Conceptual representation of this work. SG receives efferent and afferent information from spinal cord (intermediolateral complex) and dorsal root ganglion, respectively, and transmits efferent information to the heart. In this work, we investigate the neural activity and its relationship to functional activity. 
D) Among 17 Yorkshire pigs, 11 had heart failure induced by ventricular pacing, and 6 were healthy controls. SG recordings were collected at terminal experiments for both groups.

E) Experimental flow.

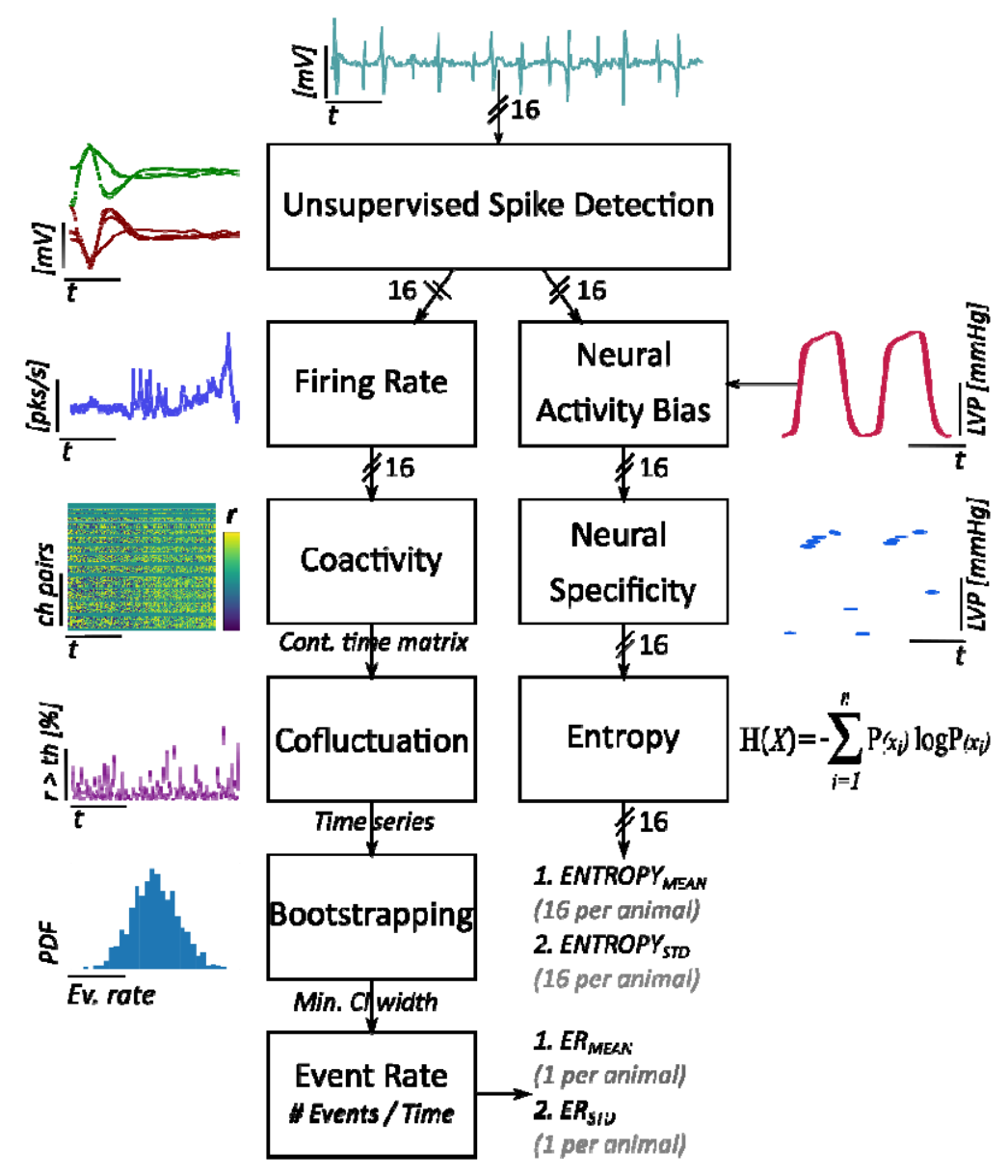

Figure 2. Signal processing block diagram. Unsupervised spike detection was applied to all LMA channels. For event rate (ER) calculation, only neural signals were used. A coactivity matrix was constructed and cofluctuation time series were bootstrapped at multiple thresholds. Final ER was based on the minimum confidence interval (CI) width of the bootstrapped ERs, individualized to each animal. For entropy estimation, LVP signal was used along with neural signals. Neural specificity and entropy time series were quantified throughout the experiment. 
Final ER and entropy values were obtained from the mean and standard deviation (STD) of parsed values of the specified interval.

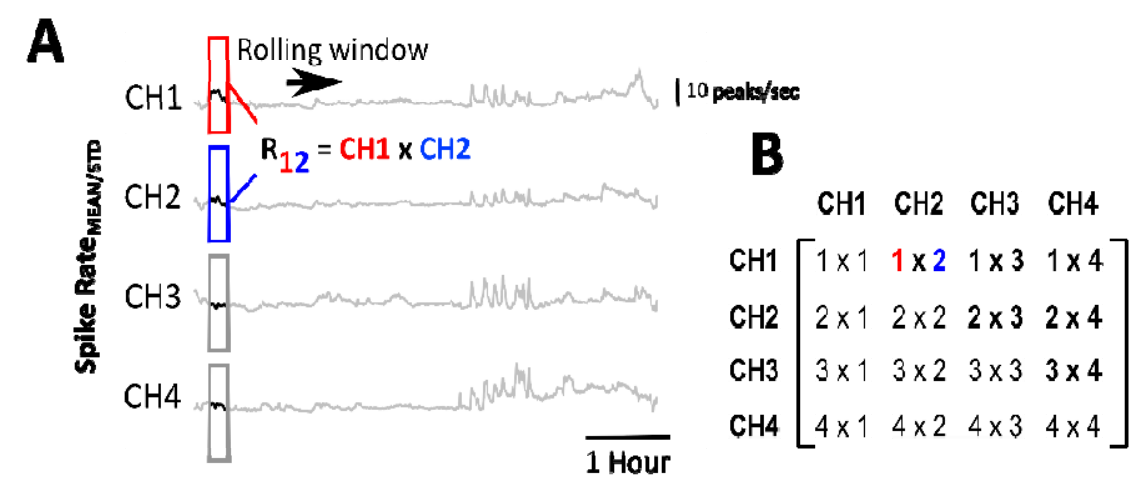

$\mathbf{C}_{\mathbf{i}}$

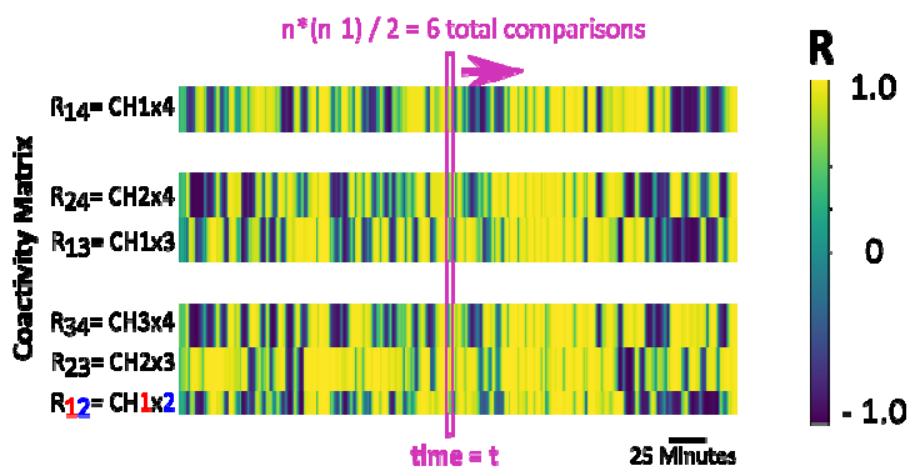

CII

25 Minutes

0

$-1.0$

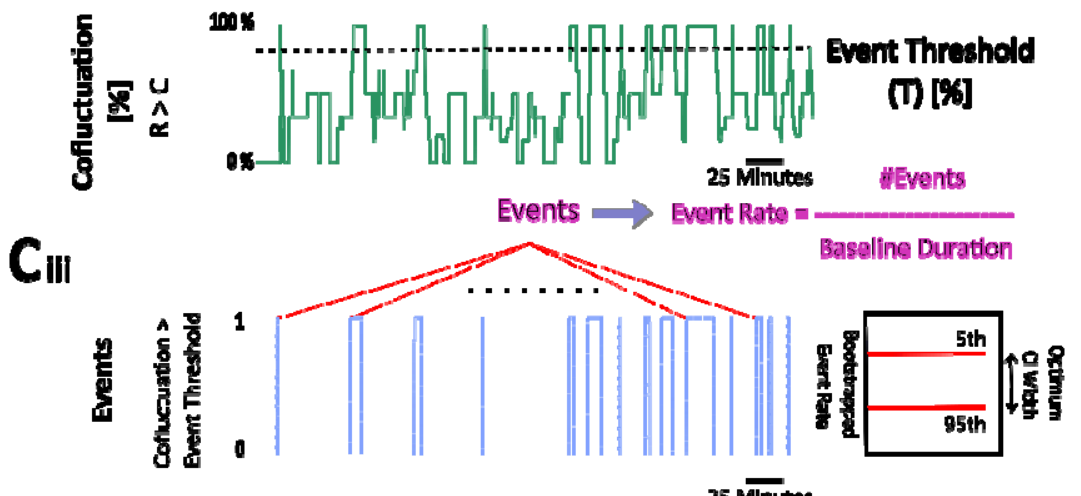

Figure 3. Coactivity and event rate (ER) computation pipeline, represented by 4-channels for simplicity.

A) Pearson's correlation coefficients $(\mathrm{R})$ obtained from pairwise rolling spike rate windows.

B) R's were inserted into the upper diagonal of a 4 x 4 matrix at a single timepoint.

C) Coactivity-time matrix, colors represent $\mathrm{R}$ value, $\mathrm{y}$ axis represent the pairwise channel pairs. At a single timepoint, a total of $n^{*}(n-1) / 2=6$ comparisons were obtained. 
D) Cofluctuation time series represent the $R$ values higher than a cofluctuation threshold (C) at a single timepoint.

E) Events time series represent the time intervals for which cofluctuations stayed at an event threshold.

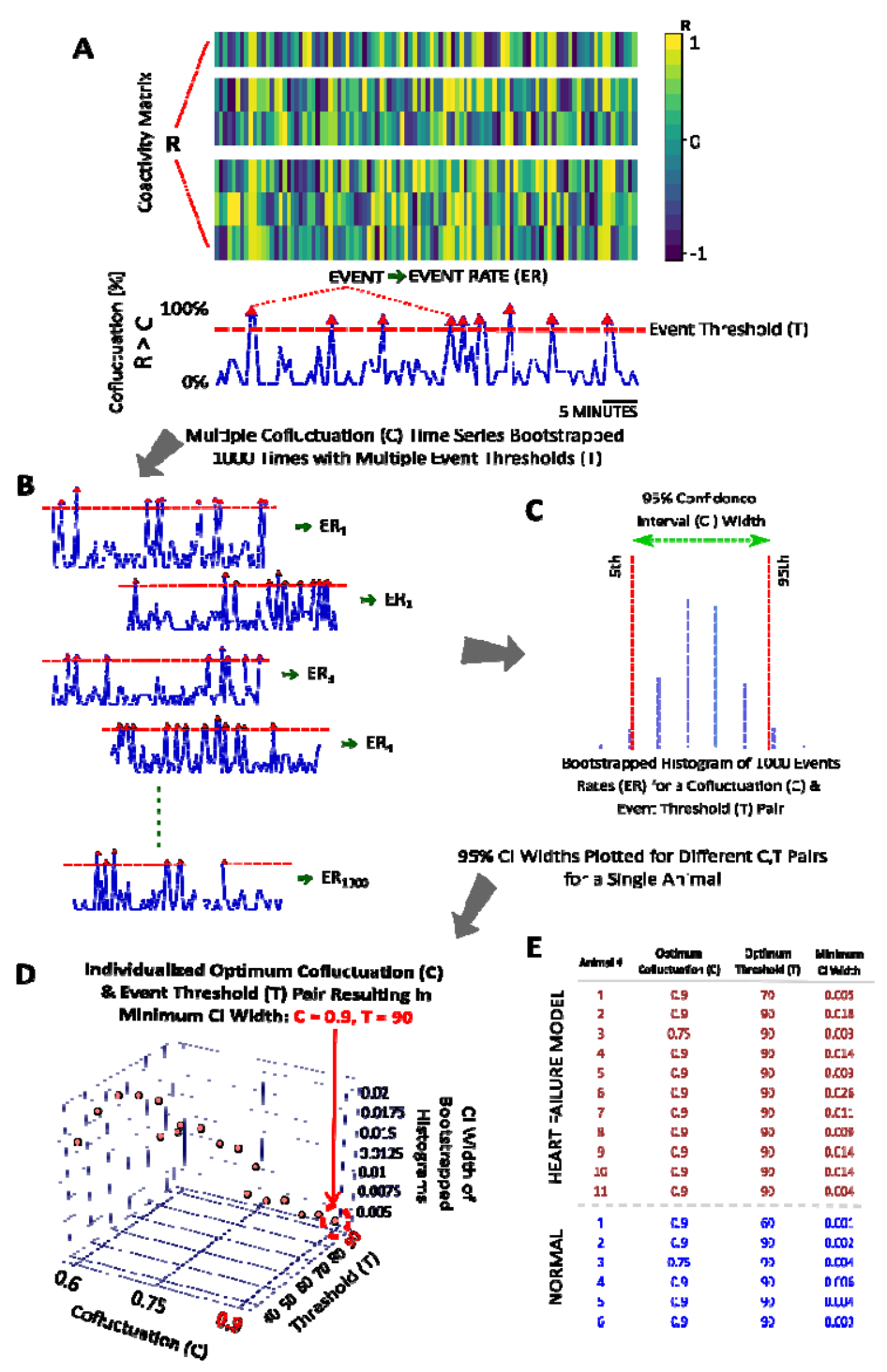

Figure 4. Cofluctuation bootstrapping pipeline for finding individualized event rate (ER) for each animal.

A) Coactivity-time matrix and cofluctuation time series at cofluctuation threshold $\mathrm{C}$ and event threshold T. 
B) For varying Cs, cofluctuation time series were bootstrapped with 1000 samples and ERs were calculated at varying Ts.

C) Bootstrapped histogram of ERs for a single C and T pair, 95\% confidence interval (CI) width was found for each pair.

D) CI width of the bootstrapped thresholds for an animal. The optimal threshold pair corresponds to the pair that results in a nonzero ER with minimum $\mathrm{CI}$ width

E) List of optimal threshold pairs and corresponding CI widths for each animal.
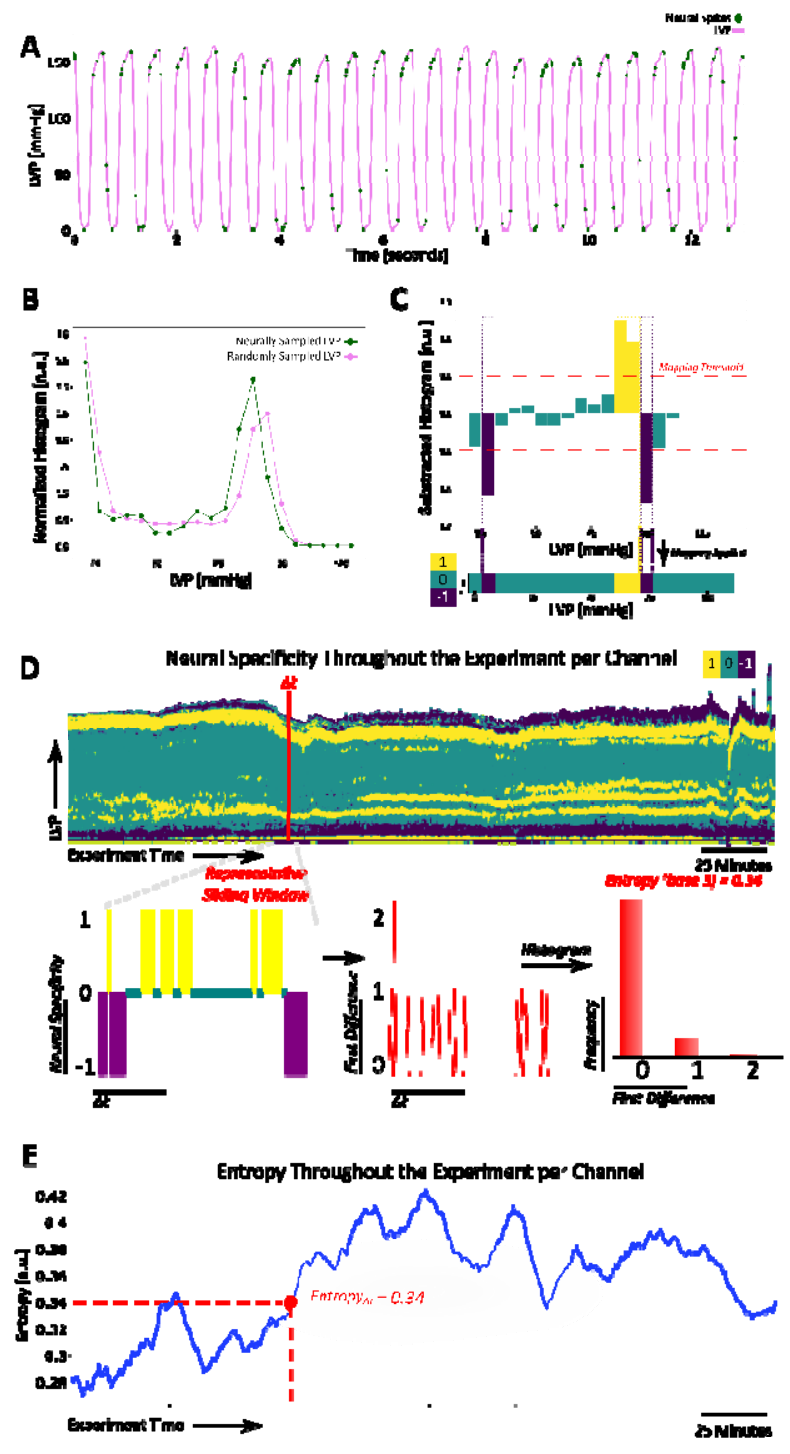

Figure 5. Neural specificity and entropy computation pipeline.

A) A short segment of data showing left ventricular pressure (LVP) and neural spikes appearing mostly phase-locked to near-peak systole of LVP.

B) Normalized histograms of random sampled LVP and neutrally sampled LVP. 
C) Bars showing subtracted histograms, colors indicating the threshold-applied data with threshold $\alpha=0.5$. Data $>+\alpha$ is shown in yellow, data $<-\alpha$ is shown in blue, data $\in(-\alpha,+\alpha)$ is shown in teal.

D) Neural specificity time series throughout the experiment per channel. At each sliding window at $\Delta t$, three mapped values are present. Entropy was estimated by finding the values of the histogram computed from the first difference of the mapped neural specificity at $\Delta \mathrm{t}$.

E) Entropy time series corresponding to the overall experiment, $\Delta$ t entropy computation in D was marked in red.
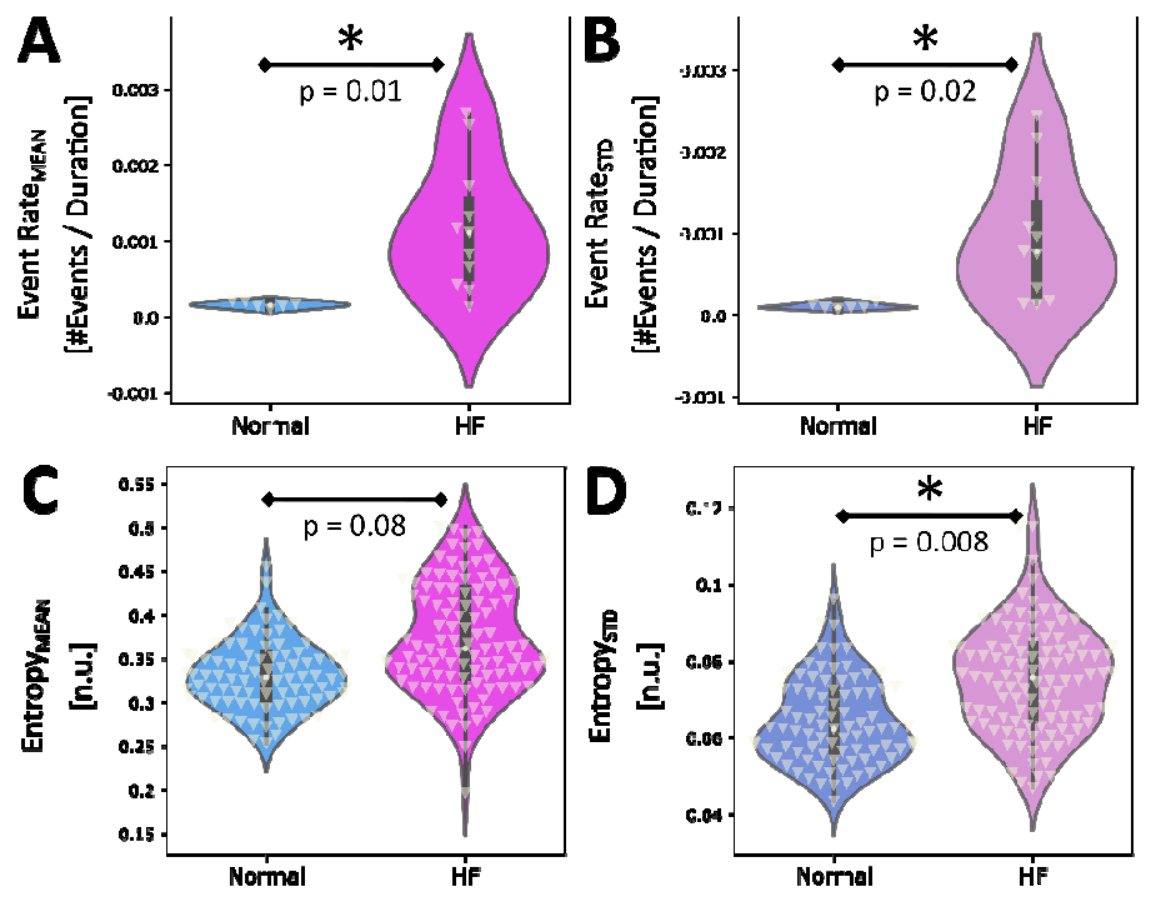

Figure 6. Event rate (ER) and entropy results for baseline interval. White triangles indicate data points.

A-B) Heart failure (HF) group animals show significantly higher ER mean and ER std compared to normal group $(\mathrm{p}<0.05)$.

C-D) HF group animals show significantly higher entropy variability ( $p=0.008)$, however no significant difference in mean entropy $(\mathrm{p}>0.05)$

For ER, p-values were calculated from two-sample t-test or Wilcoxon rank-sum tests, depending on normality. For entropy, p-values were calculated from linear mixed effects (LME) detailed in methods. 

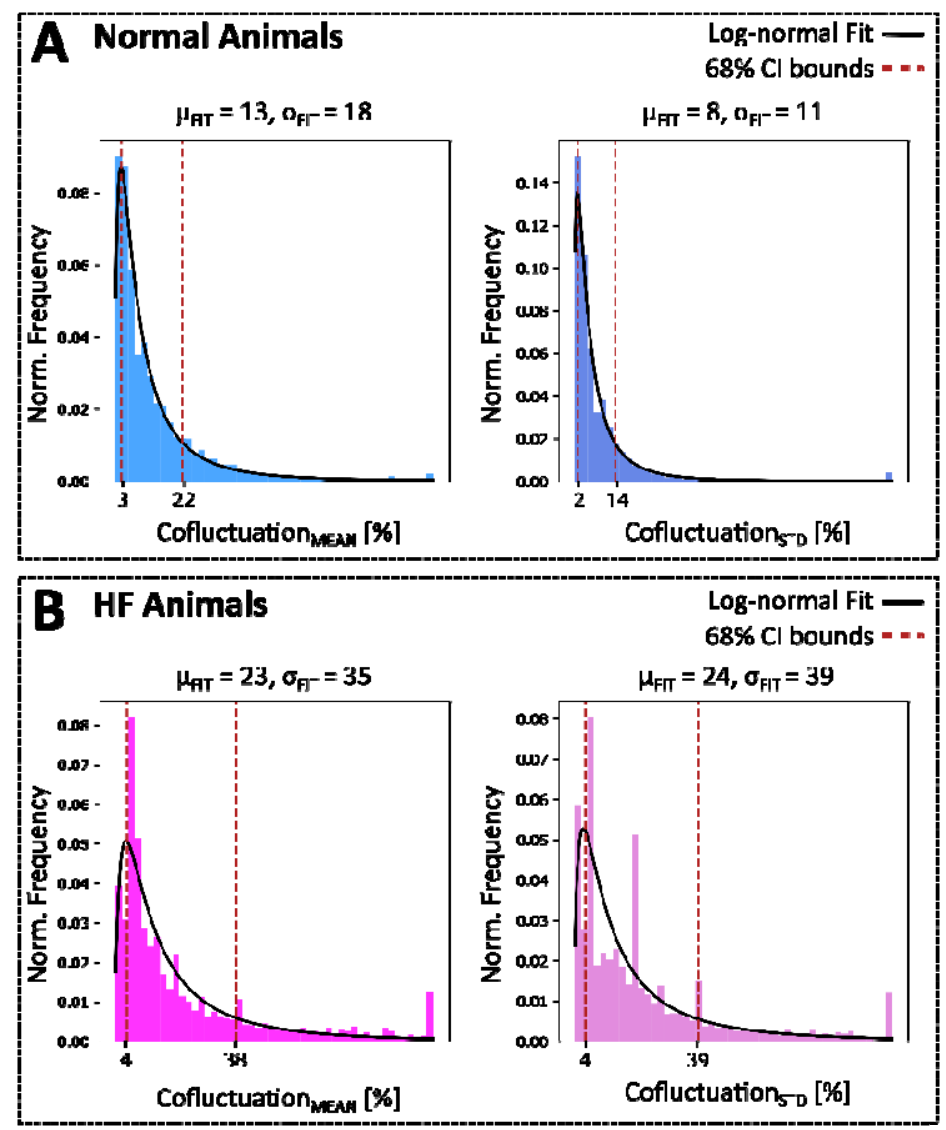

Figure 7. Cofluctuation histograms (calculated from mean or standard deviation of rolling spike rate, referred as Cofluctuation MEAN $_{\text {and Cofluctuation }}$ STD, respectively) and log-normal fits for each animal group. $\mu_{\text {FIT }}$ and $\sigma_{\text {FIT }}$ are the mean and standard deviation (STD) of fitted distribution, used for $68 \%$ confidence interval bounds.

A) Normal animals have narrower bounds and represent a better fit to log-normal distribution.

B) Heart failure (HF) animals display more heavily skewed distributions. Heavy tails are of interest for the event rate results of this work. 
A

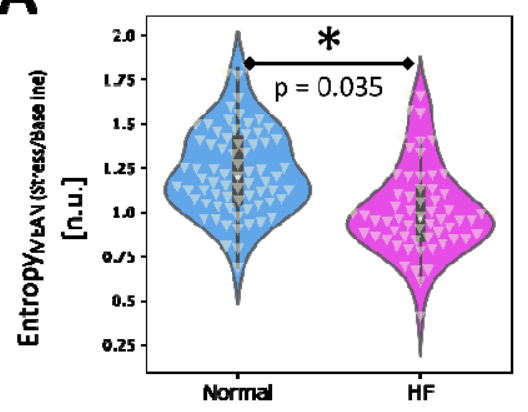

C

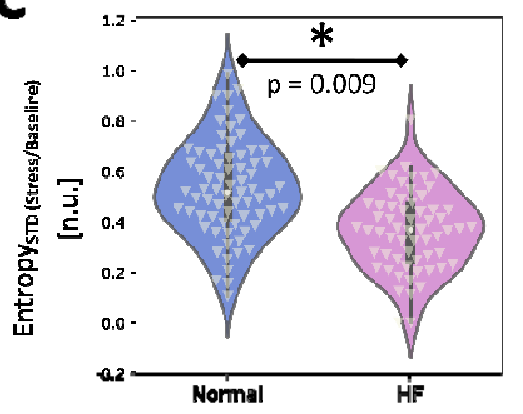

B
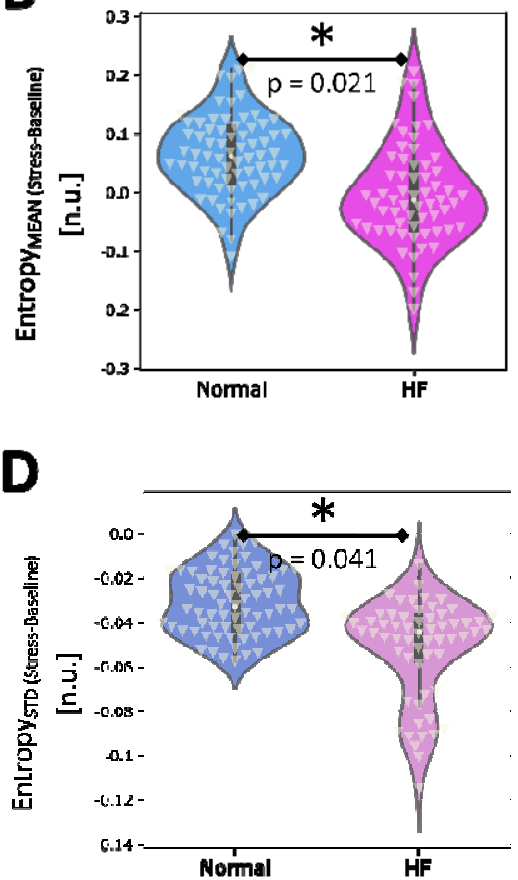

Figure 8. Entropy results for stress, stressors were averaged per channel (normalized to baseline, with either division stress/baseline or subtraction stress-baseline). White triangles indicate data points.

A) Entropymean (Stress/Baseline) (percent change from baseline).

B) Entropy meAn (Stress-Baseline) (absolute change from baseline).

C) EntropystD (Stress/Baseline) (percent change from baseline).

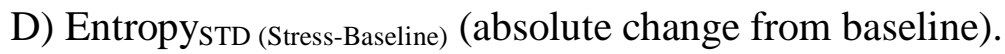

Normal animals show increase in Entropy HF animals show decrease or no change $(\mathrm{p}<0.05)$.

Both groups show decreased variability in entropy (Entropy $\left.{ }_{S T D}\right)$ during stress. The variability in entropy during stress appears significantly higher for normal animals, compared to HF.

Differences between animal groups were explored by linear mixed effects analysis. * indicates $\mathrm{p}<0.05$ for animal group (Normal/HF) based on linear mixed effects analysis. 

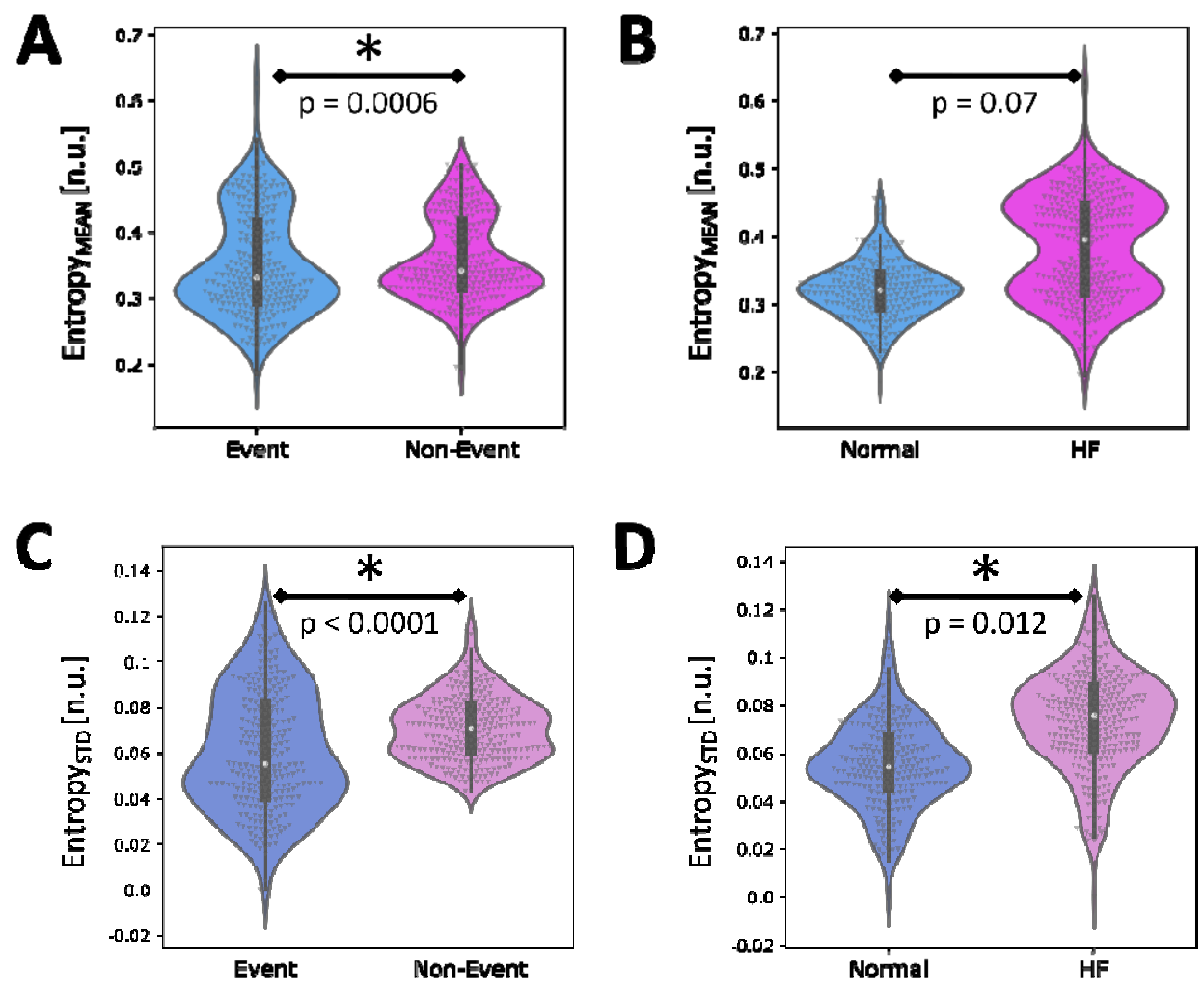

Figure 9. Event entropy investigation, entropy values were parsed in and out of events.
A) There is significant difference in EntropymEAN between events and non-events $(\mathrm{p}=0.0006)$.
B) There is no significant difference in Entropy $y_{\text {MEAN }}$ between animal groups $(\mathrm{p}=0.07)$.
C) There is significant difference in EntropystD between events and non-events $(p<0.0001)$.
D) There is significant difference in EntropystD between animal groups $(p<0.012)$. 


\section{Supplementary Material 1: Supplementary Methods}

\section{Stressor Descriptions}

After collection of baseline data, the following stressors were applied in random order:

Inferior vena cava (IVC) Occlusion: IVC occlusion decreases cardiac preload (venous return) which results in reduced left ventricular volume, cardiac output, and arterial pressure. Afferent signals from mechanosensitive cardiopulmonary and arterial baroreceptors decrease during IVC occlusion. We used a balloon catheter to temporarily occlude IVC.

Aortic Occlusion (AOC): Occlusion of the descending aorta increases cardiac afterload (arterial blood pressure) that leads to increased left ventricular pressure. Afferent signals from mechanosensitive cardiopulmonary and arterial baroreceptors are expected to increase during AOC. We used an intravascular balloon catheter to temporarily occlude the aorta downstream from the aortic arch, targeting an increase of $20 \mathrm{mmHg}$ in systolic LVP.

Decremental Right Ventricular (RV) Pacing: Various types of pacing protocols expose arrhythmogenic cardiac circuits, leading to sympatho-excitation. In decremental RV pacing, we decreased the cardiac cycle length (increased heart rate) from 450ms (133 bpm) to 250ms (240bpm) progressively by 50ms steps over 100 s using a programmed cardiac stimulator (Micropace, EP320, NSW, Australia and Prucka Cardiolab System, GE Healthcare, Chicago, IL). 
Cardiac Afferent Activation: The cardiac sympathetic afferent reflex was examined by epicardial application of bradykinin $(20 \mu \mathrm{g} / \mathrm{ml})$ and capsaicin $(20 \mu \mathrm{g} / \mathrm{ml})$ to the left ventricular anterior aspect of the heart for 30 seconds. Between each application, we waited for 30-minutes to allow for recovery of hemodynamic function.

Right Stellate Ganglion and Vagus Nerve Stimulation: Right stellate ganglion stimulation (RSGS) and right vagus nerve stimulation (RVNS) were applied as transient cardiovascular stressors. RSGS was performed at $4,8,10 \mathrm{~Hz}$ at $4 \mathrm{~ms}$ intervals for 30 seconds. RVNS was performed at $10 \mathrm{~Hz}$, in $10 \mathrm{~ms}$ intervals for 40 seconds. The output of the stimulation was set at twice threshold. Threshold was defined as the output current at which blood pressure or heart rate changed by $10 \%$ from pre-stimulus levels (increase with RSGS, decrease with RVNS).

Respiratory Stress: Mechanical ventilation was momentarily stopped, or tidal volume increased/decreased to modulate respiration dynamics, venous return, and cardiac output.

Mechanical Stimuli: A number of areas of the exposed heart (right and left ventricle) and elbows were touched mechanically to induce mechanical stress. 


\section{Supplementary Material 2: Supplementary Figures}

\section{Coactivity Matrix from Rolling Mean Spike Rate}

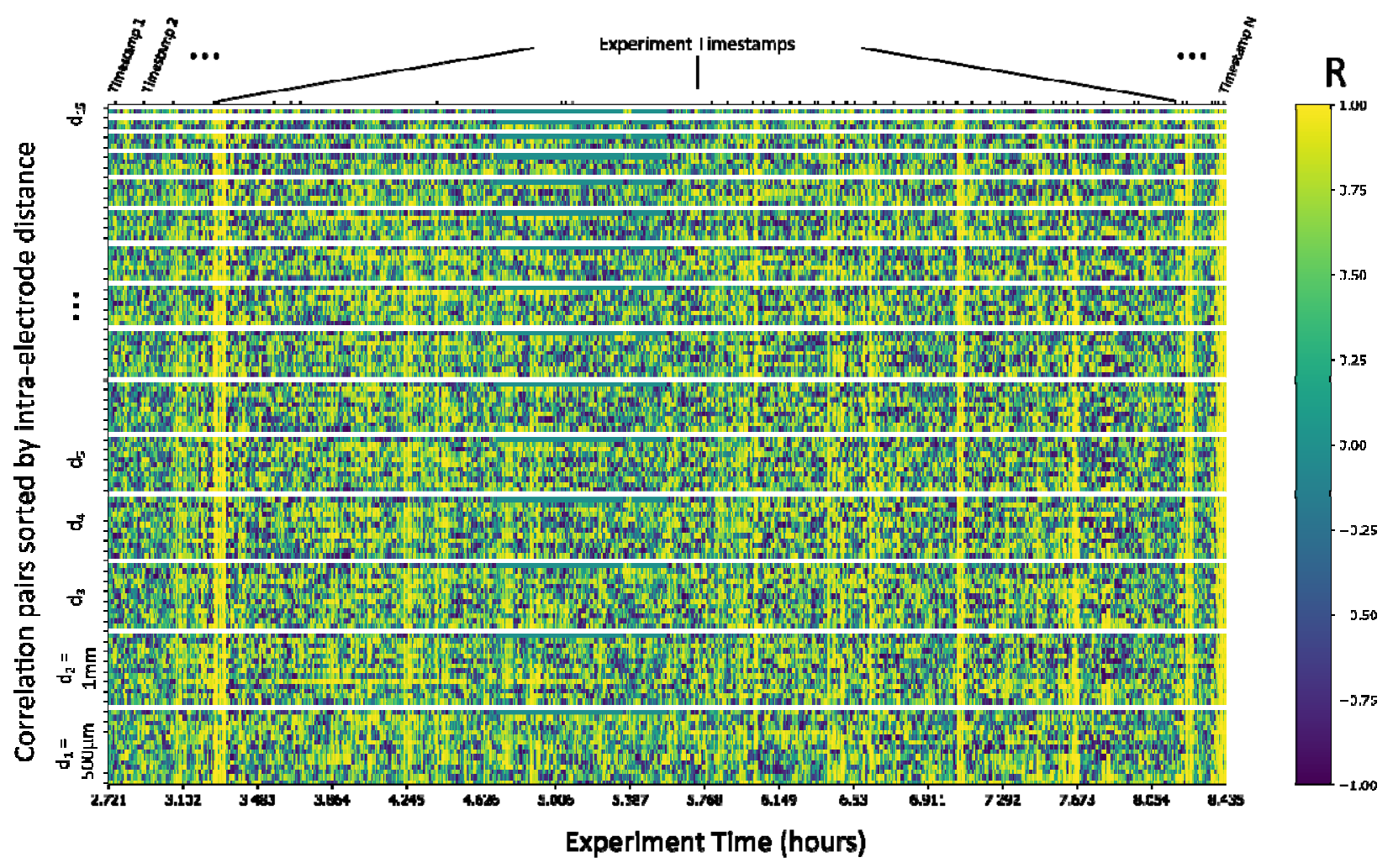

Supplementary Figure 1. A sample coactivity matrix computed from the rolling mean of spike rate for the 16-channel linear mapping array (LMA) used throughout an experiment. Y axis shows correlation pairs (i.e., Channel 1 vs. Channel 2). Correlation pairs were stacked such that the lowest row correspond to the channels separated by the minimum inter-electrode distance $\left(\mathrm{d}_{1}\right.$ $=500 \mu \mathrm{m}$, 1-electrode away), and inter-electrode distance sorted from 1 to 15 till the top. Fir instance, the highest row shows the pair separated by the maximum inter-electrode distance $\left(\mathrm{d}_{15}\right.$ $=15 * 500 \mu \mathrm{m}=7.5 \mathrm{~mm}, 15$-electrodes away). The order corresponds to the superdiagonals of the $16 \times 16$ correlation matrix. Y axis includes 120 comparisons for 16 channels, colors indicate Pearson's correlation coefficients specified in the color legend. 


\section{Events at Optimal Threshold Palrs, Case: MEAN}
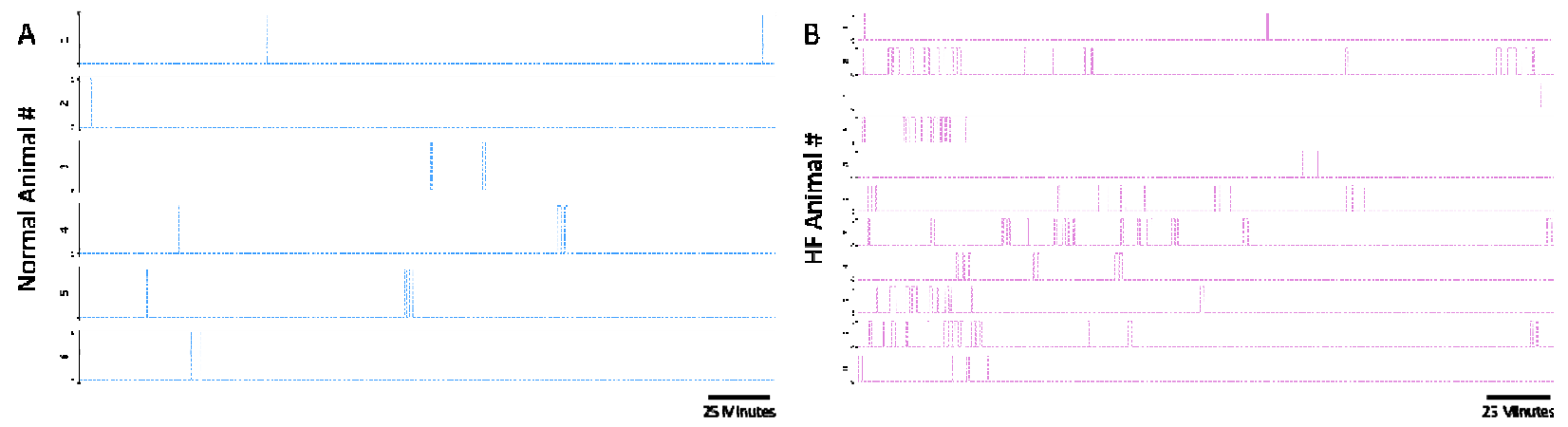

\section{Events at Optimal Threshold Pairs, Case: STD}
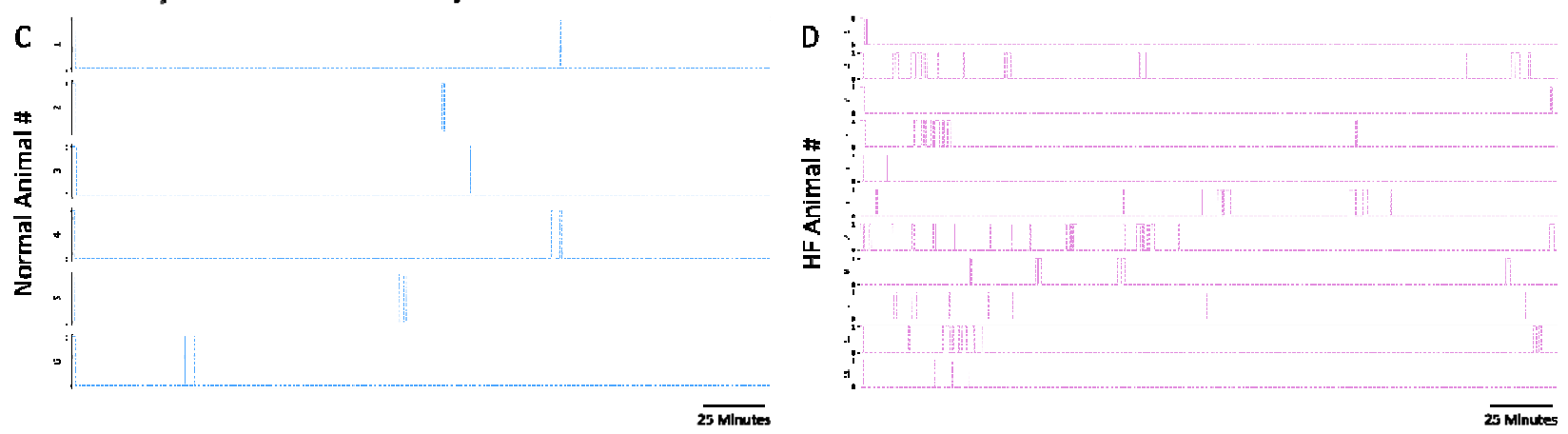

Supplementary Figure 2. Events at optimal threshold pairs for each animal.

A) Events from coactivity calculation from mean of rolling spike rate for normal animals.

B) Events from coactivity calculation from mean of rolling spike rate for heart failure (HF) animals.

C) Events from coactivity calculation from standard deviation of rolling spike rate for normal animals.

D) Events from coactivity calculation from standard deviation of rolling spike rate for $\mathrm{HF}$ animals. 

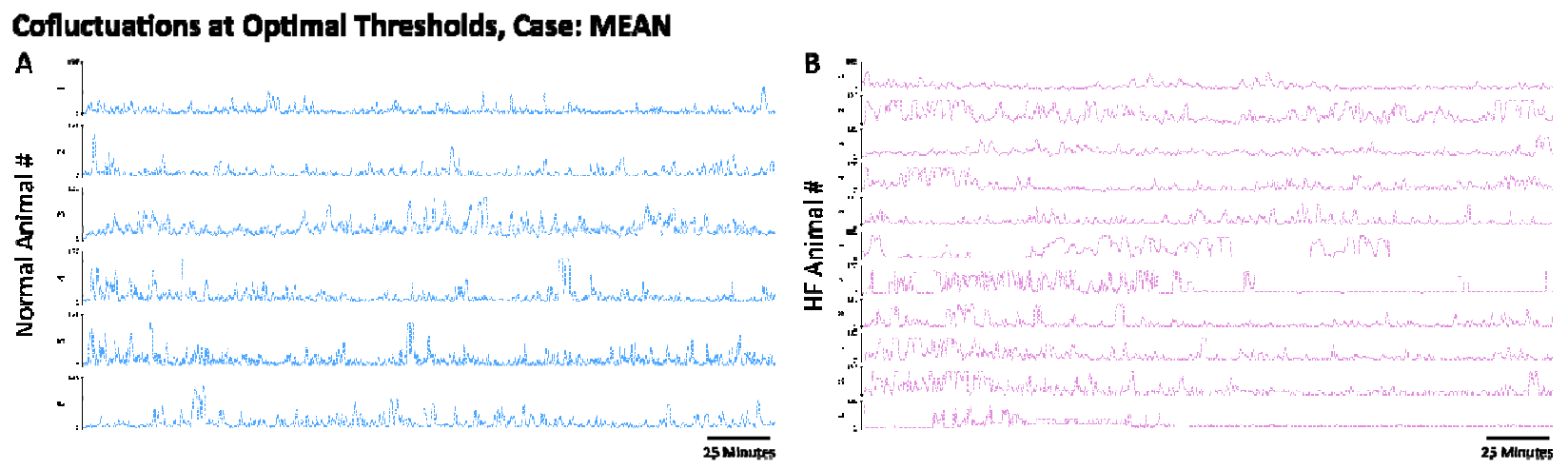

Cofluctuations at Optimal Thresholds, Case: STD
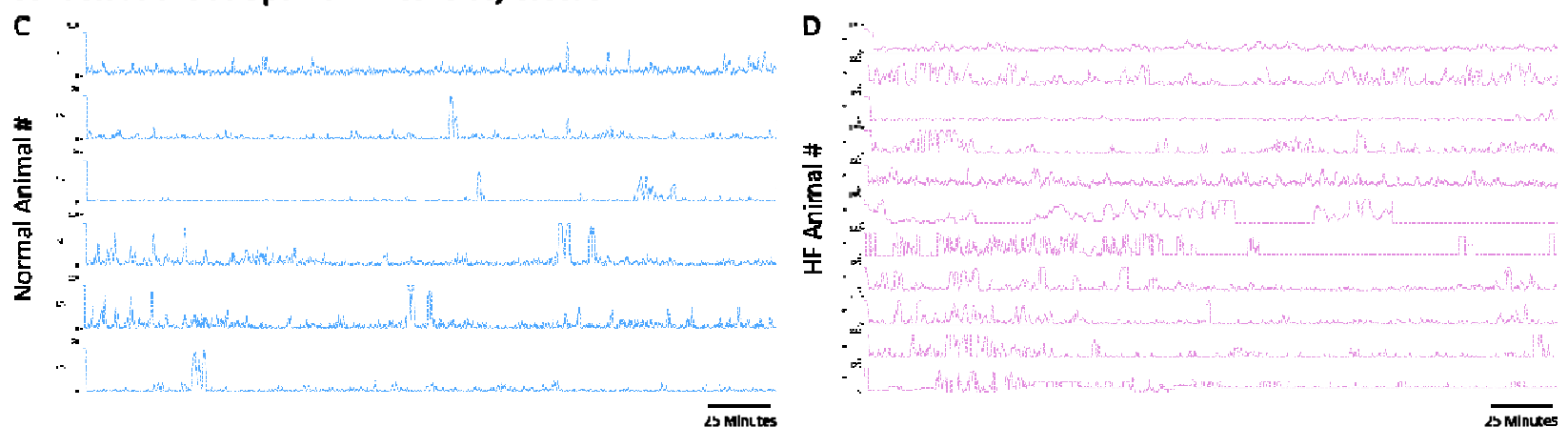

Supplementary Figure 3. Cofluctuations at optimal thresholds for each animal.

A) Cofluctuations from coactivity calculation from mean of rolling spike rate for normal animals.

B) Cofluctuations from coactivity calculation from mean of rolling spike rate for HF animals.

C) Cofluctuations from coactivity calculation from standard deviation of rolling spike rate for normal animals.

D) Cofluctuations from coactivity calculation from standard deviation of rolling spike rate for HF animals. 